\title{
A traffic-aware electric vehicle charging management system for smart cities $\boldsymbol{w}^{2}$
}

\author{
Pablo Barbecho Bautista ${ }^{a, 1, *}$, Leticia Lemus Cárdenas ${ }^{a, 2}$, Luis Urquiza Aguiar ${ }^{\mathrm{b}}$, \\ Mónica Aguilar Igartua ${ }^{a}$ \\ a Dep. of Network Engineering, Universitat Politècnica de Catalunya (UPC), Barcelona, Spain \\ b Dep. of Electronics, Telecommunication and Networks, Escuela Politécnica Nacional (EPN), Quito, Ecuador
}

\section{A R T I C L E I N F O}

\section{Article history:}

Received 13 May 2019

Received in revised form 20 August 2019

Accepted 21 September 2019

Available online $\mathrm{xxxx}$

\section{Keywords:}

VANET

Electric vehicles (EVs)

Smart charging service

Vehicle-to-vehicle

Applications

Intelligent transportation systems

\begin{abstract}
A B S T R A C $T$
The expected increase in the number of electric vehicles (EVs) in the coming years will contribute to reducing $\mathrm{CO}_{2}$ pollution in our cities. Currently, EVs' users may suffer from distress due to long charging service times and overloaded charging stations (CSs). Critical traffic conditions (e.g., traffic jams) affect EVs' trip time (TT) towards CSs and thus influence the total trip duration. With this concern, Intelligent transport systems (ITS) and more specifically connected vehicle technologies, can leverage an efficient real-time EV charging service by jointly considering CSs status and traffic conditions in the city. In this work, we propose a scheme to manage EVs' charging planning, focusing on the selection of a CS for the energy-requiring EV. The proposed scheme considers anticipated charging slots reservations performed through a vehicular ad hoc network (VANET), which has been regarded as a cost-efficient communication framework. In specific, we consider two aspects: 1) the EV's total trip time towards its destination considering an intermediate charging at each candidate CS, and 2) the communication delay of the VANET routing protocol. First, in order to estimate the EV's total trip time, our CS selection scheme takes into account the average road speed, traffic lights, and route distance, along the path of the EV. The optimal CS that produces the minimum total charging service time (including the TT) is suggested to that energyrequiring EV. Then, we introduce two communication modes based on geographical routing protocols for VANETs to attain an anticipated charging slot reservation. Simulation results show that with our charging scheme EVs' charging service time is reduced and more EVs are successfully charged.
\end{abstract}

(C) 2019 Elsevier Inc. All rights reserved.

\section{Introduction}

Now a day, developed countries are implementing policies to reduce carbon emissions and promote sustainable transportation. Among many innovative solutions, electric vehicles (EVs) are considered the most promising alternative to reduce carbon dioxide greenhouse emissions in the transportation sector [1]. In this context, The European Union has set one of the most challenging

* This work was partly supported by the Spanish Government through projects TEC2014-54335-C4-1-R (INcident monitoRing In Smart COmmunities, INRISCO) and TEC2017-84197-C4-3-R (Secure sMArt Grid using Open Source Intelligence, MAGOS).

* Corresponding author.

E-mail addresses: pablo.barbecho@upc.edu (P. Barbecho Bautista), leticia.lemus@upc.edu (L. Lemus Cárdenas), luis.urquiza@epn.edu.ec

(L. Urquiza Aguiar), monica.aguilar@upc.edu (M. Aguilar Igartua).

1 Is recipient of a Ph.D. grant from Secretaria Nacional de Educación Superior, Ciencia y Tecnología (SENESCYT), Ecuador.

2 Is recipient of a Ph.D. grant from the Academic Coordination of the University of Guadalajara, Mexico. targets for reducing carbon dioxide greenhouse emissions from vehicles in the world. By the year 2021, $\mathrm{CO}_{2}$ emissions from new vehicles are expected to be $42 \%$ less than new vehicles in 2005 [2]. USA, China, Japan, Canada, among others, lead the rapid development of the EVs' industry. The decisive shift of drivers to use EVs is contributing to further reductions in $\mathrm{CO}_{2}$ emissions. Since EVs are expected to continue growing, a new problem arises for drivers regarding where to charge. In general, EV's battery charging is a time-consuming process, although fast-charging stations and EVs capabilities for different types of charging levels help to reduce charging time [3] [4]. Battery swapping technology is another option for discharged batteries in EVs [5]. It reduces substantially time at charging stations compared to the plug-in or inductive charging. However, battery-charging stations will be the most widely method to recharge EVs because battery swapping needs a more complex infrastructure. Therefore, to optimally manage where to charge has become an important research problem in recent years because the relatively long time needed to charge an EV battery. Furthermore, in case of several EVs are planning 
to charge at the same CS (e.g., the CS is located at a highly visited place) it may become overloaded rapidly, which decreases the quality of experience (QoE) of the EV's user.

Most of the current research works in this topic investigate the EV charging management in terms of energy-scheduling at the CS, but they are not concerned about EVs' mobility [6] [7]. In this regard, challenges include the selection of an appropriate CS for the EV during its driving phase (i.e., during the EVs' journey), and the design of a communication framework to support the EV charging service.

In this paper, we first introduce a CS-selection scheme aims to minimize the total trip time of the user. Here, we consider traffic conditions on the EV's route towards its destination taking into account an intermediate recharging stop at the selected CS, thus minimizing the total trip duration.

Second, we propose a communication framework among EVs and RSUs to interchange charging service messages. The framework should be highly dynamic and flexible to support the EV charging service and not interfere to road safety services for smart cities.

To have inter-vehicle communications (IVC), wireless technologies such as traditional cellular technologies (i.e., long term evolution LTE), cellular vehicle-to-everything (C-V2X) or IEEE 802.11p could be used in our CS-selection approach. LTE and C-V2X may significantly increase the communication cost in terms of signaling and delay, since both need a higher overhead than VANETs [8]. Moreover, LTE communications need the use of base station infrastructure and employ a licensed band. Nevertheless, the use of cellular networks for the EV charging service would be provided to customers by subscription, which could include an additional cost for users. Alternatively, a cost-efficient solution is the use of VANETs which can provide high data transfers and low communication latency. Here, vehicles can operate to forward service messages to other vehicles or to road side units (RSUs) located along the city. In this way, cooperative behavior among vehicles allows EV drivers to reduce their charging service time. Furthermore, such a cooperative scheme would assist the smart grid (SG) to efficiently manage a centralized charging slots reservation scheme in the CSs deployed throughout the city. Hence, our communication framework is based on two geographical routing protocols of vehicular ad hoc networks (VANETs). We also evaluate another communication scheme named opportunistic. Specifically, we analyze the store-carry-forward paradigm, where EVs buffer and forward messages opportunistically, valid for delay-tolerant applications in vehicular delay tolerant networks (VDTNs) [9]. It is worth noting that a similar cooperative, decentralized approach can be implemented with LTE device-to-device technology [10].

Finally, we carry out extensive simulations under realistic urban scenarios to validate the effectiveness of our proposed EV charging management system. Here, the proposed strategy clearly outperforms the most current charging system today (i.e., noncoordinated).

In a nutshell, we carefully design an efficient EV charging service for urban areas when EVs with a critical state of charge (SoC), i.e., insufficient battery level to complete the trip, will require charging during their trip. We take advantage of IVC so that EVs can communicate with the city infrastructure and with other vehicles to perform an anticipated charging slot reservation. This feature will definitively improve the efficiency of the EV charging service.

The rest of the paper is organized as follows. Related work is discussed in Section 2. Then, the proposed system is presented in Section 3 including the EVs' trip time estimation and CS-selection strategy. After that, Section 4 details the service message interaction and the service data pakets. In Section 5, we introduce two communication schemes based on geographic routing protocols for EVs-RSU communications, and also on an opportunistic commu- nication scheme. Next, Section 6 shows the benefits of our EVs charging management scheme in realistic urban environments. Finally, conclusions and some future work are drawn in Section 7.

\section{Related work}

The increasing EVs penetration that we are witnessing today motivate that several works study the impact of EVs in the power grid (e.g., voltage deviations, transformers overloading) [11]. Most of the literature, such as [12-15], focuses on optimizing the energy-scheduling coming from either fossil fuels or renewable energy resources. Most of these works consider that the EV is already at an CS or parked at home; thus, EVs' mobility issues in the charging management is neglected. Just a few works like [16], [17] consider the charging service required during the EV's trip.

The proposed strategies for charging management in the literature can be classified into (i) centralized and (ii) decentralized schemes.

(i) A central intelligence or global controller manages EVs' charging. This controller considers the smart grid (SG) state and the CSs' state (e.g., available energy). Here a communication framework is required so that the energy-demanding EVs communicate with the global controller. The benefits of using a centralized coordination scheme can be found in [18-20]. Among those benefits, we highlight adaptive real-time operation and better utilization of resources, since a central management unit might consider the current global state of the EVs' batteries and the amount of available energy in the SG devoted to EVs. In [21], authors present a centralized charging management scheme where CSs are selected by considering the electricity price.

(ii) In a distributed scheme, EVs select an CS based on their local knowledge and on each driver's personal preference. The optimal $\mathrm{CS}_{\text {opt, EV }}$ selection (i.e., CS with the minimal charging time) is not guaranteed but the communication framework is less complex. In [22], authors propose a decentralized EV charging management scheme, where CSs disseminate their available time for charging (i.e., waiting time till start their charging service). EVs use this information to select an appropriate CS. Here, the system efficiency depends on the CS dissemination frequency. In [23], an EV charging management scheme based on communications between EV-RSU and EV-CS is presented. The former is a vehicle-to-infrastructure (V2I) communication, whereas the latter is a vehicle-to-grid (V2G) communication. Here, authors evaluate different VANET routing protocols in terms of end-to-end delay. Their results show the impact of choosing a routing protocol, which clearly affects the average charging service time.

Nevertheless, the above literature does not consider the current traffic conditions in the city, nor the impact of the communication protocol in the EV charging framework. Thus, the actual charging service time may increase, since the EVs' trip time (TT) increases under dense traffic conditions, which typically happen in urban scenarios. Moreover, the EVs' charging reservations may be affected by traffic conditions. For instance, a reservation may prematurely expire before the EV reach the reserved slot in the planned CS. Very few works undertake the issue of traffic conditions during the energy-demanding EV's trip towards the intended CS. In [24], the drivers' trip duration and mobility uncertainty (e.g., a traffic jam) are considered for selecting an CS. In that work, the EV periodically sends a service request to a centralized controller through a cellular network, which responses with the CS selected for that EV. Alternatively to those works proposed in the literature, we present a communication framework based on VANETs with a novel CSselection scheme for an efficient centralized traffic-aware scheme to manage the EVs charging system. 


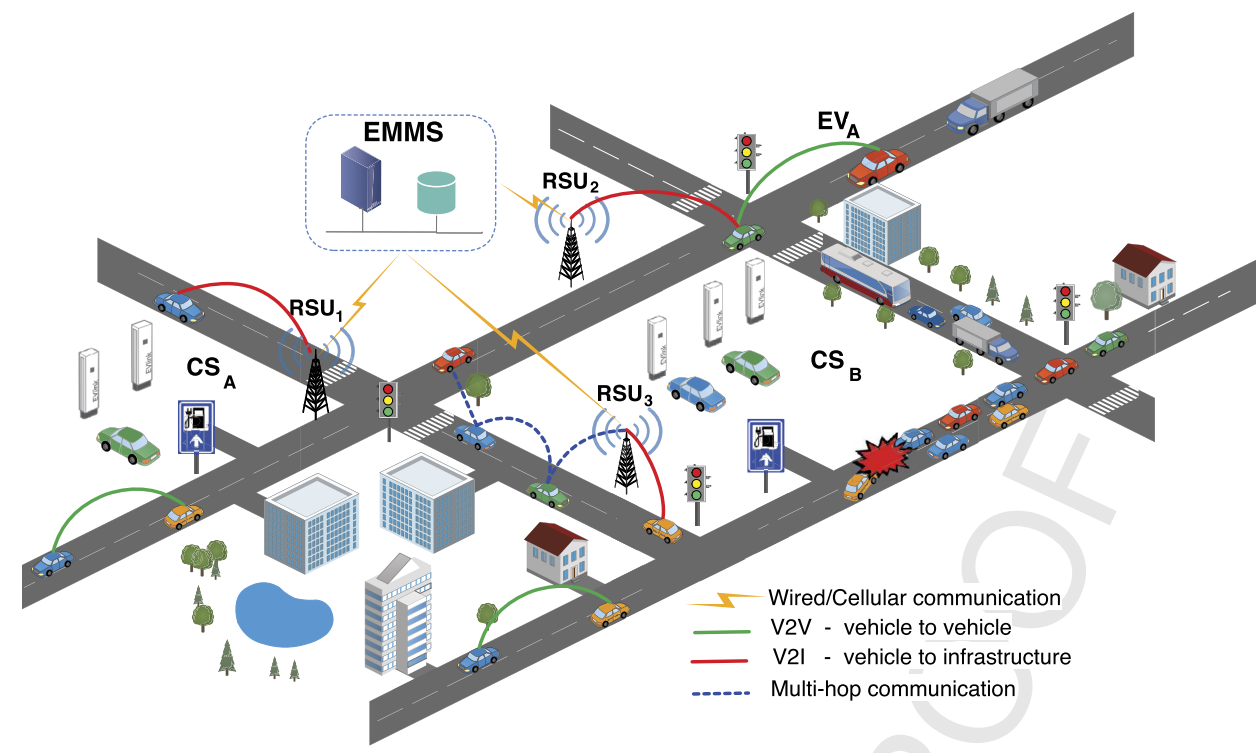

Fig. 1. Overview of the proposed EV charging management system. (For interpretation of the colors in the figure(s), the reader is referred to the web version of this article.)

\section{Electric vehicle charging management system}

As we said, most reservation-based charging systems, consider fixed reserve expiration times generally oversized (e.g., reserve expires in 1 hour). In this way, those systems aim not to consider traffic conditions or the occurrence of unforeseen situations that may increase the total trip duration towards the selected CS. However, when a large number of EVs plan to charge at the same charging station, it can get congested rapidly, reducing the users' QoE. Under that situation, EVs could select a non-optimal CS.

To cope with this, we claim that it is essential to consider the trip duration on the EVs' charging planning. Specifically, our goal is to minimize the total trip time to destination for each EV. Thus, when we detect that an EV has a low battery below a given threshold, in case it does not have enough battery to get destination the system will look for a proper CS to charge the battery. Current traffic conditions are taken into account seeking to reserve the CS where the EV will arrive sooner.

\subsection{Definition of entities of the system}

1. EV: Each EV appears in the scenario with a certain trip destination. As soon as the EV moves, it progressively discharges its battery. When an EV presents a low battery state of charge (SoC) during its trip, meaning that its battery is below a threshold, the EV starts to negotiate with the EMMS to find a suitable CS for charging.

Further to this, the EV confirm the reserve of the charging slot through the nearest RSU (either directly, see a red line in Fig. 1 or according to a multi-hop process, see dotted blue lines in Fig. 1).

2. CS: Each CS communicates their local status (e.g., electricity needs of EVs, electricity availability in the CS) to the EMMS. CSs are able to charge EVs in parallel, based on multiple charging slots.

3. RSU: RSUs are located at strategic positions within the city, including all CSs, to provide effective and reliable communications with the city infrastructure. The RSUs allow the communication between EVs and EMMS.

4. EMMS: It consists of a centralized entity in charge of the EV's charging planning. The electro-mobility management server (EMMS) has a holistic view of EVs and CSs conditions within the charging service area (i.e., the city).
5. Network: In order to plan EVs charging, we propose to use VANETs, as an alternative to cellular communications. It is well known that infrastructureless VANETs can offer low latency due to short distances, compared to traditional cellular communications. Nonetheless, the promising C-V2X [25] technology also allows direct communication between vehicles, thus without incurring the long latency present in cellular networks in which messages have to pass through access points. Therefore, our proposal for EVs charging management scheme could easily be adapted as well to C-V2X.

To forward the service messages used in our proposal of an EV charging system, we consider three geographic routing protocols, which are described in Section 5 and evaluated in Section 6 .

In this paper, we assume the following:

(i) Each vehicle is equipped with a global position system (GPS) as well as with an on-board unit (OBU) which allow vehicles to establish vehicle-to-everything (V2X) communications. Besides, vehicles are aware of smart city services via road-side units (RSUs) deployed along the city.

(ii) Charging stations (e.g., see $\mathrm{CS}_{\mathrm{A}}$ and $\mathrm{CS}_{\mathrm{B}}$ in Fig. 1) and RSUs are connected to the backbone network as well as to the EMMS.

(iii) Traffic conditions are locally available in the system either gathered directly from the network itself (e.g., a VANET) or from any external traffic service (e.g., Google Maps).

\subsection{System design}

In this section, we detail the main features of our proposal. An overview is shown in Fig. 1.

According to our proposal, a charging slot is intended to be reserved in the most suitable CS. The criterion is to select the CS where the EV driver experiences the minimum service time. This service time jointly considers the charging time at candidate $C S_{n}$ and the total trip time to destination including an intermediate stop at $C S_{n}$. Thus, whenever an EV needs to charge its battery, our scheme tries to find and reserve (during a specific period) the best available charging slot. In Fig. 2, our proposed system to manage the EVs' charging is shown. Notations are introduced in Table 1. The system logic is as follows: 


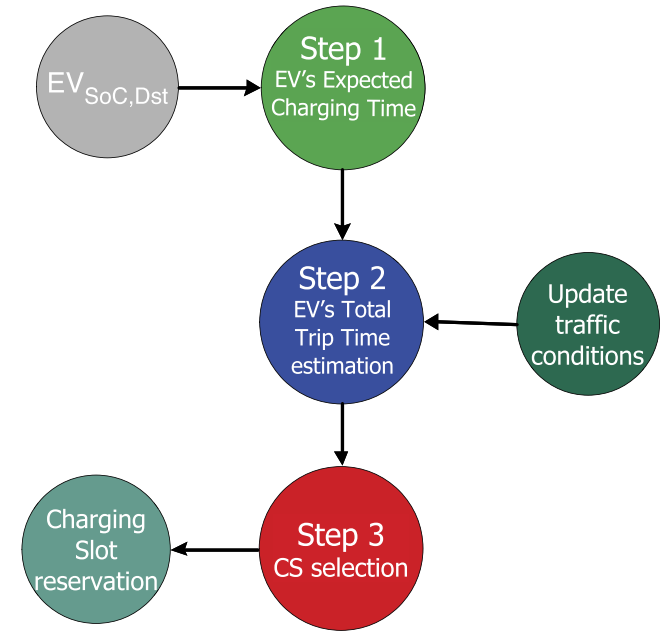

Fig. 2. Proposed system logic for the EV's charging planning.

Table 1

List of variables used in the system.

\begin{tabular}{|c|c|}
\hline Parameter & Parameter definition \\
\hline$N$ & Total number of CSs \\
\hline$A R S_{E V}$ & Average road speed \\
\hline$C S_{n}$ & Candidate charging station \\
\hline$C S_{L I S T}^{E V}$ & List of CSs attainable by the EV \\
\hline $\operatorname{slot}_{\text {LIST }}$ & List of charging slots \\
\hline$C S_{\text {opt }, E V}$ & optimal CS selected for that EV \\
\hline$C S_{\text {power }}$ & CS charging power \\
\hline slotnum $_{C S_{n}}$ & Number of charging slots in $C S_{n}$ \\
\hline slot $_{\text {selected }, C S_{o p t, E V}}$ & Charging slot reserved in the $C S_{o p t, E V}$ \\
\hline$\delta_{F F V+E V}$ & Total vehicles' density \\
\hline$\eta_{E V}$ & EV's energy consumption coefficient \\
\hline$D D_{E V}$ & EV's driving distance \\
\hline$E V_{S o C}$ & EV's current SoC (\%) \\
\hline$E V_{\text {max_cap }}$ & Maximum capacity of the EV's battery \\
\hline So $C_{\text {Threshold }}$ & $\begin{array}{l}\text { Minimum battery level to trigger a } \\
\text { charging request (\%) }\end{array}$ \\
\hline$E V_{C R}$ & EV that reports a charging request \\
\hline$E C_{C S_{n}}$ & Energy consumption to reach $C S_{n}$ \\
\hline$E V_{D s t}$ & EV's destination \\
\hline$C T_{C S_{n}}^{E V}$ & EV's expected charging time at $C S_{n}$ \\
\hline$R_{E V_{\text {curr }}, C S_{n}}$ & shortest route from $E V_{\text {curr }}$ to $C S_{n}$ \\
\hline$R_{C S_{n}, E V_{D s t}}$ & shortest route from $C S_{n}$ to $E V_{D s t}$ \\
\hline$S T_{C S_{n}}^{E V_{D s}}$ & EV's charging service time with regard to $C S_{n}$ \\
\hline$T T_{C S_{n}}^{E V_{D s t}}$ & $\begin{array}{l}\text { EV's total trip time to destination } \\
\text { including a charging stop at } C S_{n}\end{array}$ \\
\hline$T L_{E V}$ & Number of traffic lights in the EV's route \\
\hline
\end{tabular}

Step 1: First, whenever an EV needs to charge its battery we first check if the EV has enough battery to reach its intended destination (e.g., home, working place). In such a case, the EV goes there directly. Conversely, in case the EV does not have enough battery to reach its destination, it will need an intermediate stop for charging at the most suitable CS. Here, based on the EV's current location and its available $\mathrm{SoC}$, the charging time is estimated for each candidate $C S_{n}$. Candidates $C S_{n}$ are those CSs which can be reached by the EV with its current SoC.

Step 2: Based on the output of step 1, the EV's total trip time to destination is estimated, including an intermediate charging stop at each candidate $C S_{n}$. We highlight the fact that this total trip time to destination is estimated considering current traffic conditions in the scenario.

Step 3: Finally, outputs of step 1 and step 2 are used for the CS decision and reserving a charging slot at the optimal CS.

\subsection{Temporal estimations used to select the optimal CS}

As stated previously, we assume that traffic conditions are locally available, either from the vehicular network itself or from an external traffic service. Current real-time traffic reporting services and route planners (e.g., Google Maps) could be used in our scheme to estimate the EV's arrival time towards a specific CS. To mimic the actual route plan of vehicles in our simulations, we analyze which are the elements along the route that vehicles will find (e.g., number of traffic lights, intersections, the speed limit of streets). Those elements have an impact in the EVs' trip time. To characterize a trip time estimation model for urban environments, we have used a multiple linear regression to attain our traffic-aware proposal of a charging service for EVs. It is worth to highlight that this paper focuses on the influence of considering traffic conditions on the EVs' charging planning, and not on the design of a traffic reporting service or of a new route planner.

We denote as $S T_{C S_{n}}^{E V_{\text {Dst }}}$ to the service time interval from the moment when the energy-requiring $\mathrm{EV}$ reports its charging request till the moment when the EV arrives to its destination. This service time interval includes the charging time at candidate $C S_{n}$, where $1 \leq n \leq N$, being $N$ the total number of CSs.

$S T_{C S_{n}}^{E V_{D s t}}=S R T_{E V}+T T_{C S_{n}}^{E V_{D s t}}+C T_{C S_{n}}^{E V}$

Here, $S R T_{E V}$ is the service response time measured from the moment when the EV reports its charging request till the moment when the vehicle receives the service response from the network. The service response includes the location of the CS suggested where the EV is intended to be charged. Note that $S R T_{E V}$ mainly depends on the routing protocol used to forward service messages. The EV's total trip time $T T_{C S_{n}}^{E V_{D s}}$ is the time spend to travel toward the EV's destination considering an intermediate stop to charge at candidate $C S_{n}$. Note that $T T_{C S_{n}}^{E V_{D t}}$ is estimated using eq. (4) according to our linear regressions taken a wide range of representative simulation results, as it is explained in Section 3.3.2. Finally, the EV's charging time denoted by $C T_{C S_{n}}^{E V}$ means how long the EV should be stopped at candidate $C S_{n}$ during the charging phase.

\subsubsection{Expected charging time}

At the EMMS side, the expected charging time is calculated considering information reported by the energy-requiring EV. Further details of the EV's information and message exchanges are presented in Section 4.

We denote $C T_{C S_{n}}^{E V}$ as the EV's charging time at $C S_{n}$ and it is calculated as follows:

$C T_{C S_{n}}^{E V}=\frac{E V_{\text {max_cap }} \cdot\left(1-E V_{S o C}\right)}{C S_{\text {power }}}$,

where the $E V_{\text {max_cap }}$ refers to the EV's maximum battery capacity, and the $E V_{S o C}$ is the EV's current state of charge. Notice that $C T_{C S_{n}}^{E V}$ depends on the CS characteristics (e.g., fast or slow recharge). In this work, for the sake of simplicity we assume CSs are provided with enough energy and public fast-charging slots with identical charging power $C S_{\text {power }}$.

Algorithm 1, presents details regarding the calculation of the charging time. First, in case the EV's SoC is enough to reach its destination at line 4 , the EVs goes directly to its destination. Here, $D_{E V_{\text {curr }}}^{E V_{\text {s }}} \cdot \eta_{E V}$ denote the electric energy required to reach the EV driver's destination $E V_{D s t}$ based on the EV's energy consumption coefficient $\left(\eta_{E V}\right)$. On the other case, if both spare charging slots and the EV has enough energy to reach candidate $C S_{n}$ at line 8 and 10 respectively, that $C S_{n}$ is considered as reachable by the $\mathrm{EV}$. Here, $D_{C S_{n}}^{E V_{\text {curr }}} \cdot \eta_{E V}$ denote the electric energy required to reach candidate $C S_{n}$. Note that $C S_{C S_{R U S T}}^{E V}$ LIST is updated at line 11 and contains attainable CSs for the EV given its current state of charge 
$E V_{S o C}$ and position $E V_{\text {curr }}$. Finally, $C T_{C S_{n}}^{E V}$ is calculated for the energy-requiring $\mathrm{EV}$ at line 12 given (2).

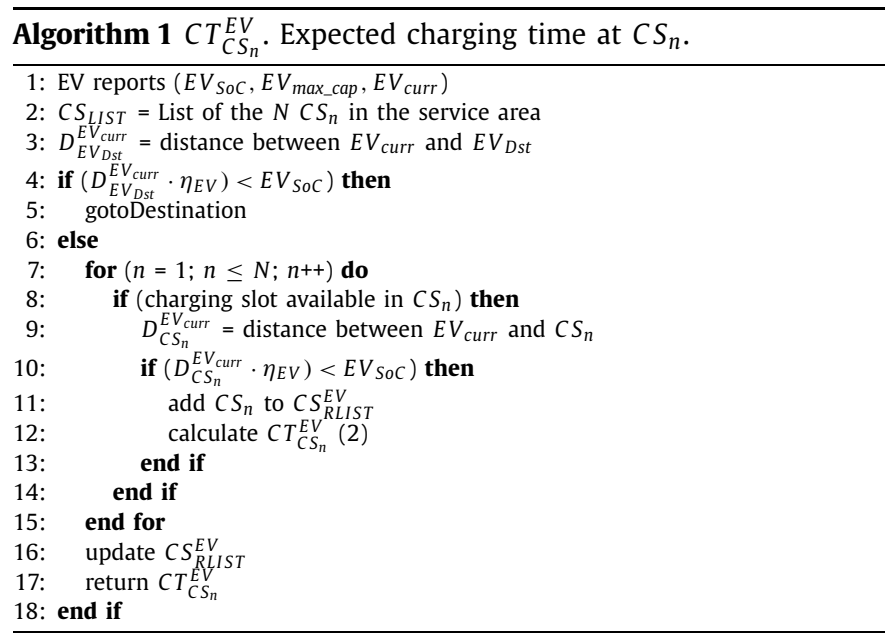

\subsubsection{Estimation of the total trip time to destination}

We denote the total trip time $T T_{C S_{n}}^{E V_{\text {Dst }}}$ as the time spent to travel toward the EV driver's destination considering an intermediate stop to charge at the candidate $C S_{n}$.

Note that considered CSs are those CSs attainable for the EV and included in $C S_{R L I S T}^{E V}$ (output from Algorithm 1). Referred to Algorithm 2, the $T T_{C S_{n}}^{E V_{D S}}$ is obtained as follows:

1. The shortest path (according to the Dijkstra's algorithm considering road map topology) toward the EV's trip destination is computed. It is composed by the shortest path toward candidate $C S_{n}$ and from that $C S_{n}$ to the $E V_{D s t}$, obtained at lines 5 and 6.

2. Upon computing EV's route, traffic conditions along the route are updated at line 7. As stated in Section 3, we assume traffic conditions are locally available gathered directly from the network (e.g., a VANET) or from any traffic service (e.g., Google Maps).

3. Then, $T T_{C S_{n}}^{E V_{\text {Dst }}}$ for the energy-requiring EV is estimated given the prediction model (4) detailed as follows. Notice that, instead of considering EV's local information (i.e., the EV's speed) for estimating its trip time, we take into consideration conditions along the route, since those will inevitably affect the total trip time (e.g., a high vehicles density reduces vehicles' speed and therefore the EVs' electricity consumption).

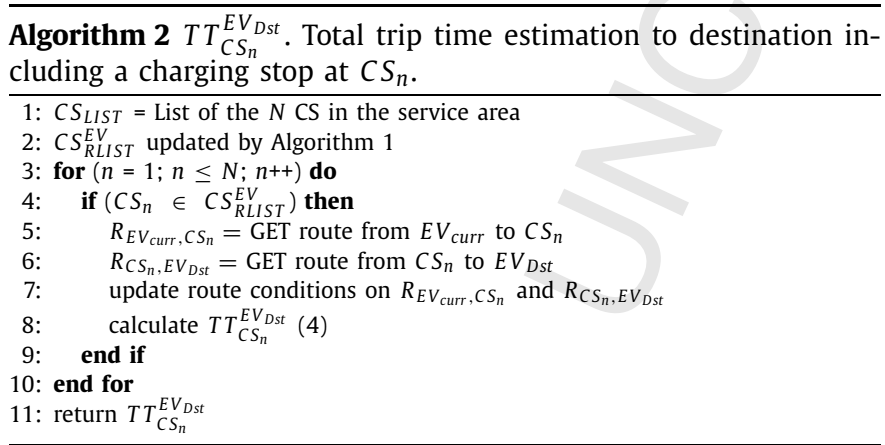

To estimate the total trip time $T T_{C S_{n}}^{E V_{D s t}}$ expressed in (4), we have characterized the additional time on route due to the presence of a certain number of traffic lights $\left(T L_{E V}\right)$. Also, we have used the average road speed $\left(A R S_{E V}\right)$ and the driving distance $\left(D D_{E V}\right)$. This way, we estimate the EV's total trip time with respect to $E V_{D s t}$ and $C S_{n}$, i.e. with respect the EV's destination $\left(E V_{D s t}\right)$ given a charging stop at charge station $C S_{n}$. Then, $T T_{C S_{n}}^{E V_{D s t}}$ is estimated as a linear function of three terms:

(a) The number of traffic lights $\left(T L_{E V}\right)$ along the EV's route towards $E V_{D s t}$ considering an intermediate charging stop at $C S_{n}$.

(b) The average road speed $\left(A R S_{E V}\right)$, calculated by averaging the road's speed $A R S_{r}$ of those roads $r$ that compose the path driven by EV. It basically depends on the current vehicles' density on those roads.

(c) The driving distance $\left(D D_{E V}\right)$ calculated by adding road distances over the map topology.

Notice that $A R S_{E V}$ could be estimated from diverse ways. For instance, we assume that smart traffic lights average consecutive speeds $\left(v_{s}\right)$ taken from vehicles' beacons through a given road $(r)$ by using an exponential weighted moving average (EWMA), see (3), where $i$ stands for the iteration index of the averaging process and $w$ (we used $w=0.25$ ) is the weight to average new ARS samples:

$A R S_{r, i}=w \cdot v_{s, i}+(1-w) \cdot A R S_{r, i-1}$

To model the total trip time $T T_{C S_{n}}^{E V_{\text {Dst }}}$ spent by an EV to travel towards destination considering a potential intermediate stop to charge its battery at $C S_{n}$, we use a multiple regression statistical tool [26] and the statistical software SPSS [27]. We have considered many representative simulations for generic urban scenarios of different dimensions and different vehicles' densities. The $T T_{C S_{n}}^{E V_{D s t}}$ is calculated as follows:

$$
T T_{C S_{n}}^{E V_{D s t}}=\alpha_{1}+\alpha_{2} \cdot T L_{E V_{D s t}, C S_{n}}+
$$

$\alpha_{3} \cdot A R S_{E V_{D s t}, C S_{n}}+\alpha_{4} \cdot D D_{E V_{D s t}, C S_{n}}$

where $\alpha_{1}$ reflects the average trip time in the assessed scenario; $\alpha_{2}$ denotes the time spend due to traffic lights found along the route. This value is derived from the traffic light cycle; $\alpha_{3}$ considers the average road speed variation; the last term $\alpha_{4}$ adds time to the $T T_{C S_{n}}^{E V_{\text {Dst }}}$ per each meter traveled in the path.

As expected, an increment on the number of TL or DD in the path represents an increase in the trip duration, whereas an increase in $A R S_{E V_{D s t}, C S_{n}}$ decreases the total trip time. Notice that $\alpha_{i}$ coefficients of the model in (4) depend on the road map, although our considered scenarios are general enough for urban environments under diverse vehicles' densities. In a future work we plan to consider a self-adaptive model that implements machine learning techniques to dynamically configure the coefficients of the model.

\subsubsection{CS selection}

The CS selection scheme looks for the CS through which the EV will experience the shortest service time $S T_{C S_{n}}^{E V_{D s}}$ (1), mainly settled by the charging time $C T_{C S_{n}}^{E V}$ and the total trip time $T T_{C S_{n}}^{E V_{D s t}}$ By running Algorithm 3, the optimal $C S_{o p t, E V}$ is selected by jointly considering the total trip time $T T_{C S_{n}}^{E V_{\text {Dst }}}$ towards the EV's trip destination with an intermediate parking duration for charging $C T_{C S_{n}}^{E V}$ at candidate $C S_{n}$. Note that the total trip time towards each candidate $C S_{n}$ is estimated taking into account current traffic conditions along the route as these affect the total trip time and therefore the CS selection. Here, a sub-optimal CS selection may diminish the EV's user QoE.

We point out that although in this work we consider the service time as metric to choose the optimal CS $\left(C S_{o p t, E V}\right)$, additional metrics could also be included as inputs in Algorithm 3. Examples 


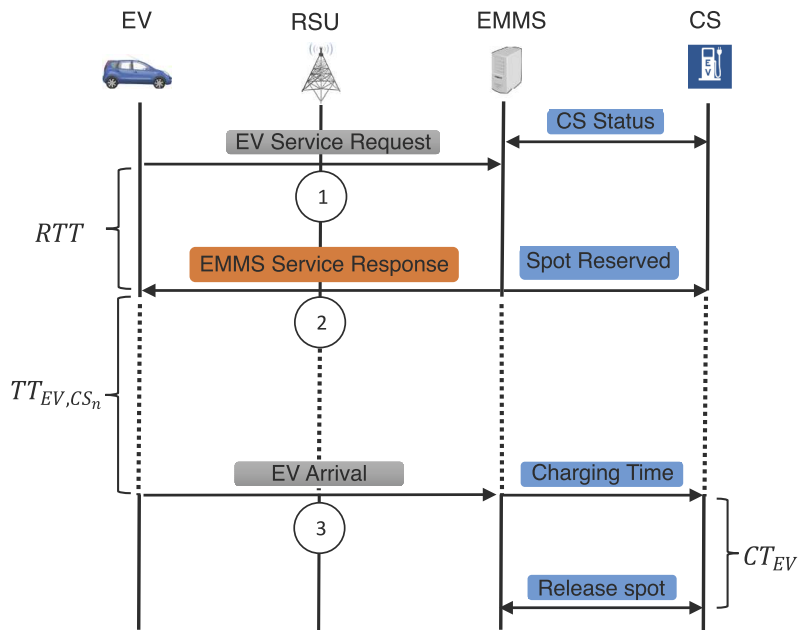

Fig. 3. Interchange of messages in the proposed EV's charging management system.

of interesting metrics to be considered are waiting time at the CS, energy price, or origin of energy (renewable or non-renewable).

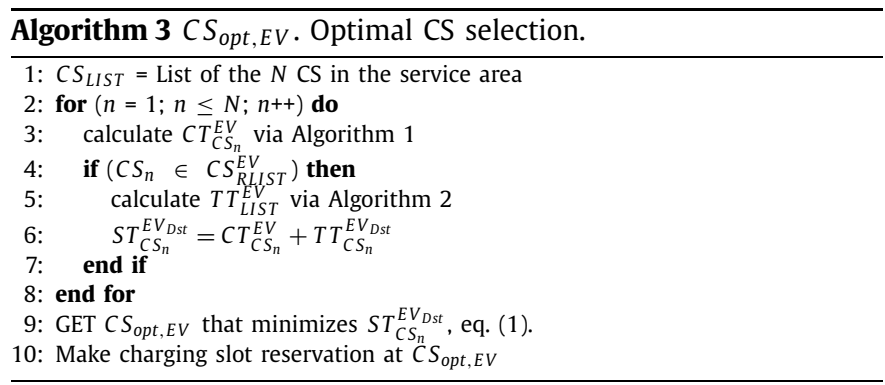

\section{Interchange of service messages}

In this Section, we detail the service messages exchange between EVs and the EMMS. The message interaction flow for the proposed charging service is presented in Fig. 3. We focus on the EV-RSU communication for an EV charging service during the EV journey (i.e., while the EV is moving). The EV-RSU communication is modeled based on the IEEE 1609 WAVE standard [28]. Considered service messages (1-3 in Fig. 3) are reported following the standard frame format detailed in the IEEE 1609 WAVE/WAVE Short Message (WSM) [29].

Once an EV notices a low battery $\left(E V_{S o C}<S o C_{\text {Threshold }}\right)$, it reports its charge requirements using the service request message to the closest RSU through the VANET (see 1 in Fig. 3). Details of the fields of the EV's request message are given in Table 2 .

At the EMMS side, the most suitable CS for the EV is selected according to Algorithm 3. Then, the EMMS responds to the EV with a service response message (see 2 in Fig. 3) which contains information of the selected CS. Table 3 gives details of the EMMS service response message. Here, the msgID field contains the application identifier. The CSinfo field contains information of the selected CS, e.g. the CS identification, the charging slot reserved for the EV.

The Service_Status field indicates to the energy-requiring EV which is the CS decision (i.e., reservation accepted or reservation request expired). The TT field contains the moment when the EV is expected to arrive at the selected CS suggested and reserved by the EMMS (i.e., it counts the EV's trip time towards the selected CS, see TT in Fig. 3).

The service messages exchange, is summarized as follows:
Table 2

EV's service information. EV service request message.

\begin{tabular}{|c|c|c|}
\hline Field & Length (Bytes) & Description \\
\hline msgID & 1 & Application ID value \\
\hline Veh_ID & 4 & Vehicle identifier \\
\hline Veh_Type & 1 & Type of vehicle (EV) \\
\hline Longitud $_{E V}$ & 4 & Vehicles' geographical Long. \\
\hline Latitude $_{E V}$ & 4 & Vehicles' geographical Lat. \\
\hline Elevation $_{E V}$ & 4 & $\begin{array}{l}\text { Vehicles' elevation } \\
\text { referenced }\end{array}$ \\
\hline SoC & 1 & EV's battery state of charge \\
\hline Service_Status & 1 & $\begin{array}{l}\text { Indicates to the EMMS that } \\
\text { the charging service is } \\
\text { required }\end{array}$ \\
\hline utcTime & 10 & Time with mSec precision \\
\hline
\end{tabular}

Table 3

Charging service information. EMMS service response message.

\begin{tabular}{|c|c|c|}
\hline Field & Length (Bytes) & Description \\
\hline msgID & 1 & Application ID value \\
\hline CSinfo & 5 & $\begin{array}{l}\text { Contains information of the } \\
\text { CS and slot selected }\end{array}$ \\
\hline Longitud $_{C S}$ & 4 & CS’s geographical Long. \\
\hline Latitude $_{C S}$ & 4 & CS's geographical Lat. \\
\hline Elevation $_{C S}$ & 4 & CS's elevation referenced \\
\hline Service_Status & 2 & $\begin{array}{l}\text { Indicates the service status } \\
\text { (CS decision) }\end{array}$ \\
\hline TT & 2 & EV's trip time \\
\hline CT & 2 & Charging time \\
\hline utcTime & 10 & Time with mSec precision \\
\hline
\end{tabular}

- EV's service request: It is triggered by a low battery event at the EV side. It includes EV general information (see Table 2). The EV reports its charging service requirement to the EMMS through the closest RSU. See 1 in Fig. 3.

- EMMS service response: At the EMMS side, the EV's service request is processed and the optimal CS is selected for the EV, as it is presented in Section 3.3.3. Then, the EMMS communicates the information of the selected CS (see Table 3) to the EV. See 2 in Fig. 3.

- EV Arrival: Message sent by the EV at its arrival at the selected $\mathrm{CS}$. At this time, the $C T_{E V}$ is updated according to the current $E V_{\text {SoC }}$. See 3 in Fig. 3.

\section{Communication schemes and routing protocols}

In vehicular networks, the network topology is inherently dynamic due to the potentially high mobility of nodes (i.e., vehicles). Geographic-based routing protocols, which are based on the knowledge of the instantaneous locations of nodes, have shown a better performance in VANETs compared to topology-based routing protocols commonly used in less dynamic networks such as mobile ad hoc networks (MANETs) [30] [31]. A similar framework to ours is proposed in [32]. In that work, energy-requesting EVs choose and reserve a charging slot in an CS, autonomously and based on the periodical dissemination of CS information. EVs communicate with CSs based on opportunistic encounters with RSUs (i.e., EVs establish 1-hop communications when they reach an RSU).

Alternatively, in this work we propose an optimal centralized CS-selection scheme using two types of geographic-routing protocols for VANETs to forward service messages that are exchanged between EVs and RSUs (to communicate with the EMMS of the SG) in a multihop manner. This way, using a multihop routing scheme we attain a more efficient EV charging management system since messages are delivered faster compared to the case of only using 1-hop communications. Under this scheme, the EV's service 


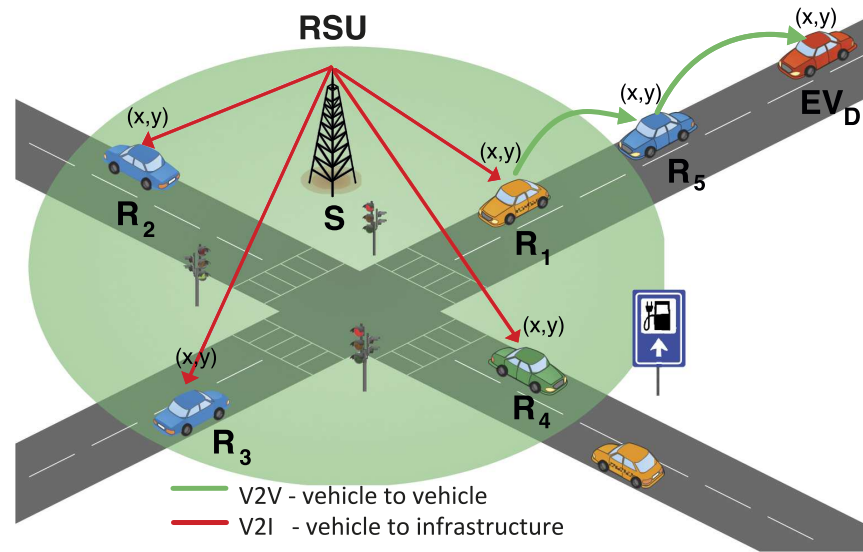

Fig. 4. Basic communication scheme for VANETs, based on message dissemination with selective forwarding.

response time $S R T_{E V}$, expressed in (1), is minimized leading to an earlier charging slot reservation.

In the following we describe the three routing protocol schemes analyzed in this work and used to forward service messages from EVs to EMMS, as well as in the reverse direction from the EMMS to the energy-requesting EVs. The three routing schemes are: (a) a simple opportunistic routing scheme with which EVs deliver messages directly to the RSUs; (b) a basic routing scheme to efficiently forward messages using a selective algorithm; and (c) an advanced routing scheme based on our previous multimetric routing proposals (MMMR) [33] and (3MRP) [34].

\subsection{Opportunistic routing scheme}

In the opportunistic routing scheme, we consider a typical store-carry-forward algorithm in which the packet travels within the vehicle instead of through the VANET. This could occasionally be done in those sparse occasions in which the current holding vehicle does not find a proper next forwarding node and during a maximum accumulated delay per packet. In this present work, we have implemented a simple opportunistic scheme as a reference to compare our two proposals explained below. In this opportunistic scheme, EVs just establish a 1-hop communication when they reach any RSU.

\subsection{Basic routing scheme}

First, we propose to use a selective forwarding mechanism for the service messages exchange in section 4 . The Basic scheme is illustrated in Fig. 4. The proposed selective forwarding mechanism reduces network overhead compared to simple flooding, seeking to avoid network congestion. It works as follows: a forwarding node broadcasts the packet if it is closer to destination than the previous forwarding node; otherwise that node refrains in the forwarding. This is repeated till the packet reaches destination. This way, the service request message sent from an energy-requesting EV the reaches the EMMS of the SG (through the nearest RSU). In this case, we assume RSUs locations are known in advance by all vehicles.

In Fig. 4, the RSU $S$ sends a response service message to the destination node $E V_{D}$ (i.e., the energy-requesting EV). Recipient nodes are those within the RSU's transmission range (nodes $R_{1}$, $R_{2}, R_{3}$, and $R_{4}$ in the example of Fig. 4). If the current receiver node $\left(R_{1}\right)$ is closer to destination $\left(E V_{D}\right)$ compared to the previous sender position $(S)$, that receiver $\left(R_{1}\right)$ decides to re-broadcast the message. The rest of intermediate receivers $\left(R_{2}, R_{3}, R_{4}\right)$, decide not to re-broadcast the message as they are farther to destination $\left(E V_{D}\right)$ than the previous sender node $(S)$.
The selective forwarding logic is described in Algorithm 4. Herein, the message includes the destination nodes' identification (ID). Once a message is received, in case the current receiver node is the destination node it processes the message (see lines 1-2); otherwise, the node computes its distance (curr_dsttoDst) with respect to the destination node (RSU or EV), see line 5 in Algorithm 4. The curr_dsttoDst is compared with the last receiver distance to destination (last_dsttoDst) included in the message (see line 6). If curr_dsttoDst is lower than last_dsttoDst then, the current node decides to re-broadcast the message since it is closer to destination (see line 8 in Algorithm 4). Before forwarding the message, the current node updates the last_dsttoDst in the message with its current closer distance to destination curr_dsttoDst (see line 7).

Notice that for the response message sent from the RSU towards the energy-requesting $\mathrm{EV}\left(\mathrm{EV}_{\mathrm{D}}\right.$ in Fig. 4), the current position of the $\mathrm{EV}_{\mathrm{D}}$ (i.e., the destination node of the response message) may have changed since the moment in which the energyrequesting message was sent from that $\mathrm{EV}_{\mathrm{D}}$ to the RSU. We estimate the $\mathrm{EV}_{\mathrm{D}}$ position in the reception moment using the former position of that EV (when it sent the query) and the trajectory of that EV (estimated from several consecutive positions gathered in the query message). Thanks to that estimation, the response message will reach the energy-requesting EV in its current position.

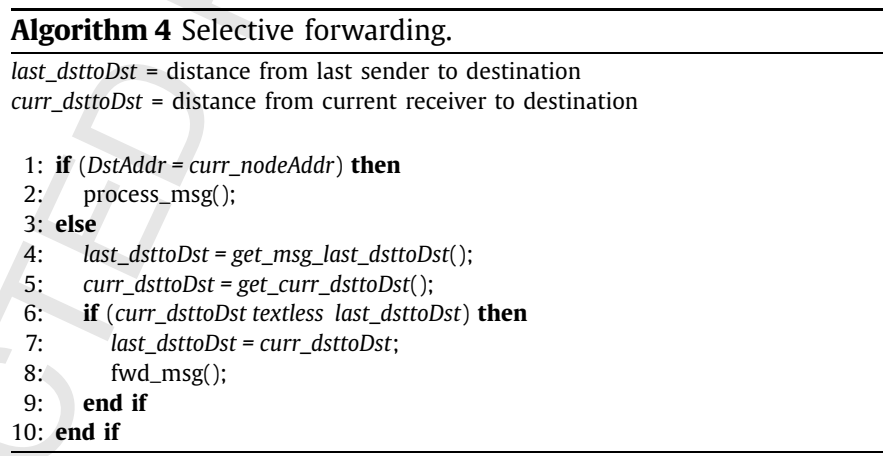

\subsection{Advanced routing scheme}

In the previous section, we have presented the basic scheme, which disseminates service messages based on a selective forwarding scheme that tries to avoid network congestion. Nonetheless, that scheme could cause significant overhead in dense scenarios due to its flooding-like operation. To cope with that, we further propose an advanced routing scheme based on our proposals named multi-metric map-aware routing protocol (MMMR) [33] and multimedia multi-metric map-aware routing protocol (3MRP) [34] for VANETs. MMMR and 3MRP are position based, traffic-aware and delay tolerant geographical routing protocols for VANETs. Both use several metrics to take hop-by-hop forwarding decisions. In [33], the authors compared MMMR against well known protocols such as greedy perimeter stateless routing (GPSR) [35] and I-GPSR (Improvement GPSR) [36]. 3MRP [34] was specially designed to transmit video warning messages over VANETs by taking the video frame types into account. Results in [33] and [34], show that MMMR and 3MRP outperform existing solutions in terms of percentage of packet losses and average packet delay.

Since in our EV charging service, EVs and EMMS interchange text messages (see Section 4), we will use the MMMR routing protocol to transmit those messages between EVs and EMMS through the VANET. Under the Advanced scheme, illustrated in Fig. 5, each node efficiently forwards packets hop-by-hop to/from the nearest RSU through the VANET. MMMR routing protocol improves 


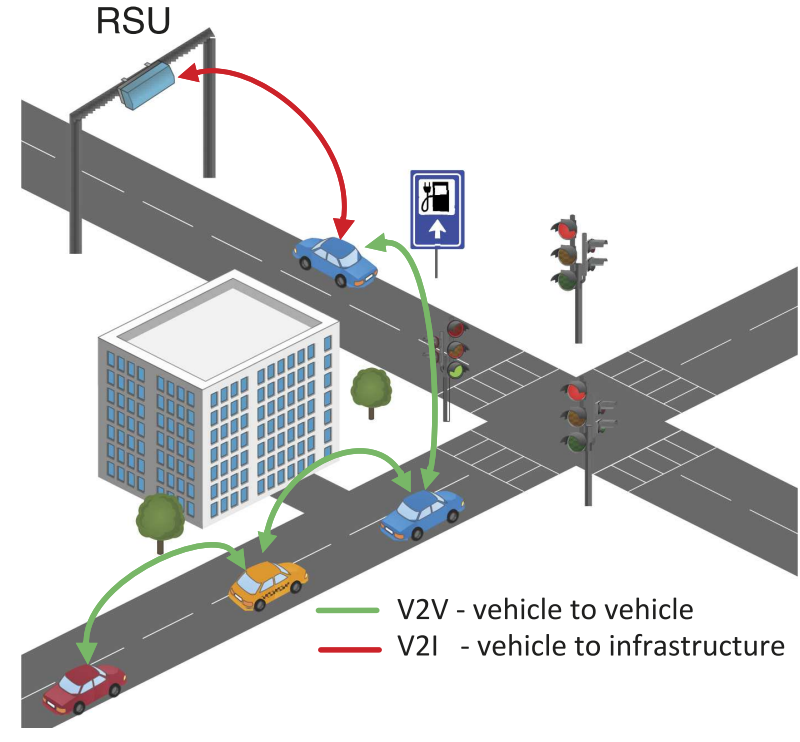

Fig. 5. Advanced communication scheme based on the MMMR [33] routing protocol for VANETs.

the next forwarding node selection based on five metrics: distance to destination, vehicles' density, vehicles' trayectory, MAC losses and available bandwidth. The MMMR multi-metric forwarding scheme minimizes the percentage of packet losses and the average packet delay. MMMR makes use of periodic beacon messages to update the neighbors nodes' information. It uses a local buffer that temporally stores a node neighbors table (NNT). The NNT is updated when a new beacon is received. If the beacon corresponds to a new neighbor, a new entry is added into the NNT. Otherwise, if the received beacon corresponds to a node that is already in the NNT, the neighbor node's parameters are updated. Each entry in the NNT becomes a candidate node to forward the packet. A multimetric value is computed for every neighbor node in the NNT that is in LoS with the current node holding the packet. MMMR forwarding decisions are performed hop-by-hop based on a multimetric score computed for each node's neighbors. This way, the multimetric calculation is able to adapt to the changing environment conditions in real time.

\section{Performance evaluation}

\subsection{Scenario configurations}

We have implemented our EVs charging management system in the open source framework VEINS [38], developed for vehicular network simulations, and OMNeT++ [39] as the network simulation platform. The electro-mobility environment (formed by EVs and CSs) is implemented using the widely-known vehicular traffic simulator SUMO [40] including buildings that may interfere the signal between sender and receiver.

Two different scenarios are considered to evaluate our proposal. On the one hand, Fig. 6a shows a medium-size dense urban scenario, with an area of $1800 \mathrm{~m} \times 1800 \mathrm{~m}$, from Barcelona city, Spain. On the other hand, Fig. $6 \mathrm{~b}$ depicts a large-size sparse urban scenario, with an area of $3200 \mathrm{~m} \times 3600 \mathrm{~m}$, from Berlin city, Germany. Road maps for each scenario include: intersections, speed limit of streets, traffic lights and buildings information imported from OpenStreetMaps (OSM) [37]. To estimate the EVs' trip duration for each simulation scenario, the $\alpha_{i}$ coefficients used in (4) and described in Section 3.3.2, are shown in Table 4.

Two types of connected vehicles are included: fossil-fuel vehicles (FFVs) and electric vehicles (EVs) with [28-50] km/h variable speed. The destination of each vehicle trip is randomly selected in the road map. EVs discharge their batteries according to the model detailed in [42]. Also, SUMO was configured with the car-following Krauss model [41], which defines a realistic car behavior with respect to vehicles on the same line of traffic flow. We consider an EVs' penetration of $10 \%, 50 \%$ and $80 \%$ with a total number of vehicles $T_{F F V S+E V s}=300$.

To set the number of charging stations (CSs), we consider EU directives described in [45], that recommended at least one public charging slot for every ten EVs on the road. Thus, for the maximum EVs' penetration percentage considered (80\%, which represents $240 \mathrm{EVs}$ ) in the network, four charging stations (CSs) are distributed within the service area with a maximum of six charging slots, see Fig. 6. We assume all CSs are fast charging points with $\mathrm{CS}_{\text {power }}=60 \mathrm{~kW}$. We also assume that the set of CSs are provided with enough energy to be able to charge the total amount of EVs present in the network.

In our simulations, EVs are moving within the service area until their batteries fall below a threshold. We set the state of charge threshold for the EV ( $\left.S o C_{\text {Threshold }}=45 \% \cdot E V_{\text {max_cap }}\right)$ to start the service request. The EV's battery size is set to $E V_{\text {max_cap }}=5 \mathrm{kWh}$. Notice this low configuration value (much lower than the current market EV's battery size) is just to speed up the battery reduction and need to charge, so that we speed up the simulation process. Actually, current EVs have $E V_{\text {batt_size }} \approx$ of $20,40,80$, or even 100 kWh [46] [47].

All the vehicles in the network are nodes of the VANET and can communicate with each other and with the city infrastructure. RSUs are located at CSs and strategically deployed in the service area, see Fig. 6. Simulations were carried out using the IEEE 802.11p standard on MAC and physical layers. The dedicated service channel SCH3 (174) is used for the energy-charging service, which is the SCH designated for road traffic efficiency in intelligent transport systems ITS [44]. We set an average transmission range of $250 \mathrm{~m}$, which is a typical value in vehicular environments. Simulation settings are summarized in Table 5 .

In Table 6 we point out the different schemes evaluated for comparison regarding the CS-selection strategy and the routing scheme used for service messages exchange between EVs and RSUs. Simulation results are presented with $95 \%$ of confidence interval $(\mathrm{CI})$ obtained from five simulations per point, each run generated with an independent seed. The following schemes are evaluated for comparison:

1. Basic traffic-aware reservation (B-TAR): The proposed CS-selection scheme with our Basic routing scheme (see Section 5.2). Here, a charging slot is reserved for the EV in the most suitable CS (where the EV arrives earlier to its destination, considering an intermediate parking duration for charging at selected CS) following a flooding-based selective forwarding mechanism for the exchange of service messages.

2. Advanced traffic-aware reservation (A-TAR): The proposed CSselection scheme with our advanced multi-metric routing scheme (see Section 5.3). Here, a charging slot is reserved for the $\mathrm{EV}$ in the most suitable CS considering an efficient service messages exchange by the means of multi-metric hop-by-hop forwarding decisions.

3. Opportunistic traffic-aware reservation $(O-T A R)$ : The proposed CSselection scheme with the opportunistic routing scheme (see Section 5.1). Unlike the previous schemes (B-TAR and A-TAR), here service packets are transmitted only when an opportunist encounter with an RSU happen. Thus, this scheme is based on 1-hop communications. That is, the packet is delivered when the EV finds an RSU. In the meantime, the EV carries the packet itself. An example that follows this scheme is [32].

4. Advanced distance-based reservation $(A-D B R)$ : This scheme looks for the closest CS (distance-based strategy), without consid- 


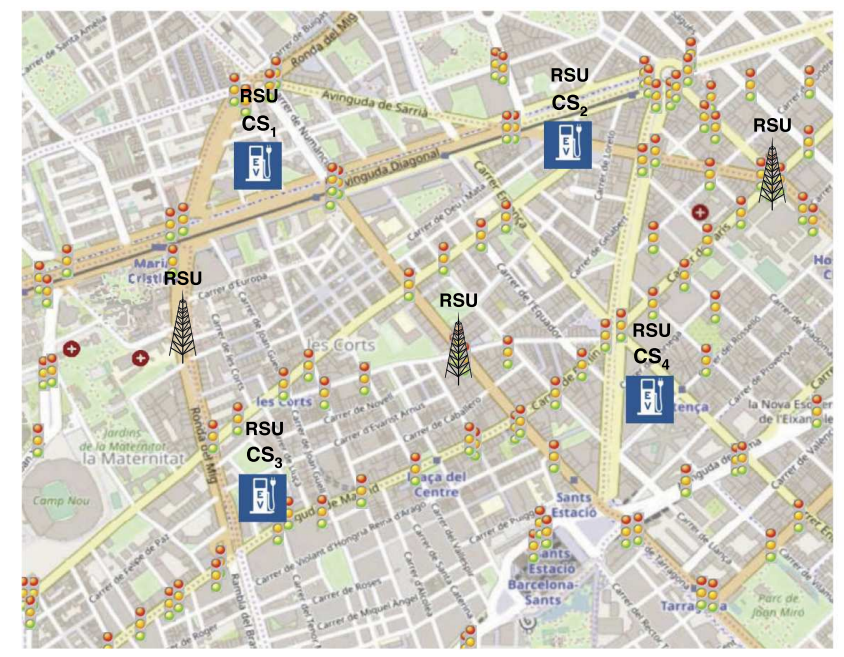

(a)

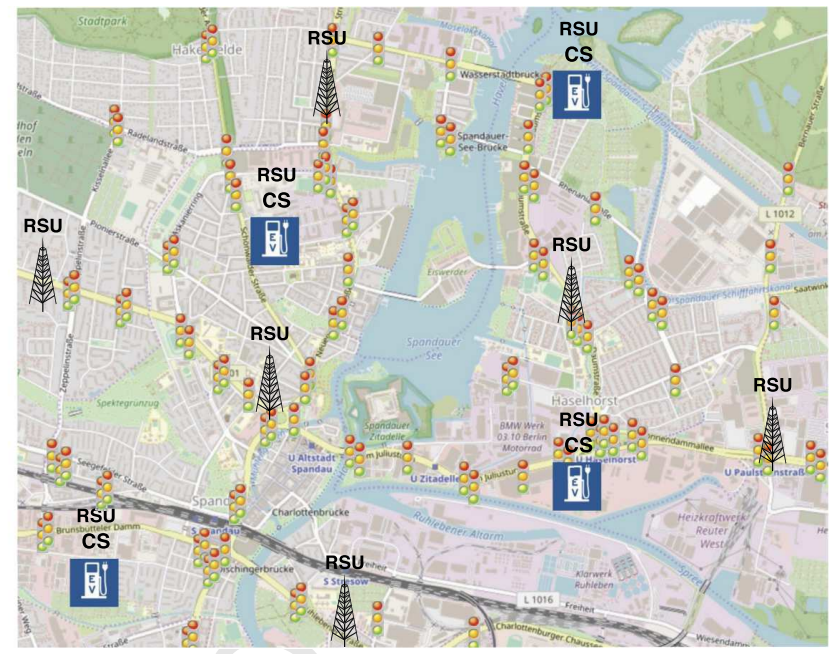

(b)

Fig. 6. Simulation scenarios considered: (a) A medium-size dense district in Barcelona, Spain; (b) A large-size sparse district in Berlin, Germany. Maps include locations of road side units (RSUs) and charging stations (CSs). Traffic lights are imported from OSM [37].

Table 4

Simulation scenario characteristics. Coefficients $\alpha_{i}$ used in (4)

\begin{tabular}{llllllll}
\hline & Intersections $/ \mathrm{km}^{2}$ & Roads $/ \mathrm{km}^{2}$ & Traffic Lights $/ \mathrm{km}^{2}$ & $\alpha_{1}$ & $\alpha_{2}$ & $\alpha_{3}$ & $\alpha_{4}$ \\
\hline Berlin & 340 & 640 & 18 & 315.8858 & 5.2640 & -18.9128 & 0.0785 \\
Barcelona & 645 & 1325 & 24 & 83.3345 & 15.4070 & -5.8474 & 0.0910 \\
\hline
\end{tabular}

Table 5

ering the traffic in the scenario. Under this scheme, the is selected based on the distance on the map (shortest path) towards each candidate $\mathrm{CS}_{n}$. Our Advanced routing scheme is configured for an efficient exchange of service messages.

5. Non-coordinated distance-based (NC-DB): This scheme represents the current situation in most cities, e.g., Barcelona. Each EV driver selects the closest CS autonomously. Thus, this is a distance-based selection scheme without reservation. If the CS selected is full at the EV's arrival, the EV goes to the next closest charging station. This scheme reflects the typical user's behavior that we can found nowadays.

\subsection{Performance evaluation metrics}

We are mainly concerned on how the system performance is affected by the increasing of EV penetration that we are lately witnessing in our cities. Accordingly, we evaluate the performance of our proposal as we increase the number of EVs in the scenario. Also, we are interested in evaluating the influence of the routing scheme used to exchange the service messages. The following performance metrics are evaluated:

- Percentage of charged EVs: The ratio between successfully charged EVs and the total number of EVs that needs to charge during the simulation time. From the city's point of view, a high percentage of charged EVs means that the charging infrastructure is better utilized.

- Average total trip time: This is the average time elapsed from the moment the EV's battery level is lower than a given threshold $\left(E V_{S o C}<S o C_{\text {Threshold }}\right)$ till the moment the EV arrives to its destination, including a potential intermediate stop to charge its battery at the selected CS. From the EV drivers' point of view, a shorter trip duration improves their QoE.

- Average electricity consumption: This is the average electricity that EVs consume given the traveled distance towards their respective destinations, considering a possible intermediate
Simulation settings.

\begin{tabular}{ll}
\hline Parameter & Value \\
\hline Map Zone & Les Corts (Barcelona) \\
Simulation area size & $1800 \mathrm{~m} \times 1800 \mathrm{~m}$ \\
Number of RSUs & 7 \\
Map Zone & Spandau (Berlin) \\
Simulation area size & $3200 \mathrm{~m} \times 3600 \mathrm{~m}$ \\
Number of RSUs & 10 \\
Vehicles' density & $60-180$ veh/km \\
EVs' penetration index & $30 \%, 50 \%, 80 \%$ \\
Mobility model & Krauss model [41] \\
EV energy model & Energy model [42] \\
EVs' battery size & $5 \mathrm{kWh}$ \\
Number of CS & 4 \\
Number of charging slots & 24 \\
Fast CS power & $60 \mathrm{~kW}$ \\
Path loss model & Empirical IEEE 802.11p [43] \\
Transmission range & $250 \mathrm{~m}$ \\
Sensing range & $\sim 300 \mathrm{~m}$ in LoS \\
Communication schemes & Opportunistic, Basic, and Advanced \\
PHY and MAC & IEEE 802.11p \\
Nominal bandwidth & $6 \mathrm{Mbps}$ \\
Service channel & SCH3 (174) for road traffic \\
& efficiency ITS [44] \\
Simulation time & $5000 \mathrm{~s}$ \\
Beacon interval & $1 \mathrm{~s}$ \\
\hline
\end{tabular}

Table 6

Operating modes to be evaluated. Our proposals are highlighted.

\begin{tabular}{lll}
\hline Mode & Routing scheme & CS-selection \\
\hline B-TAR & Basic scheme & Traffic-aware \\
A-TAR & Advanced scheme & Traffic-aware \\
O-TAR & Opportunistic scheme & Traffic-aware \\
A-DBR & Advanced scheme & Distance-based \\
NC-DB & - & Distance-based \\
\hline
\end{tabular}




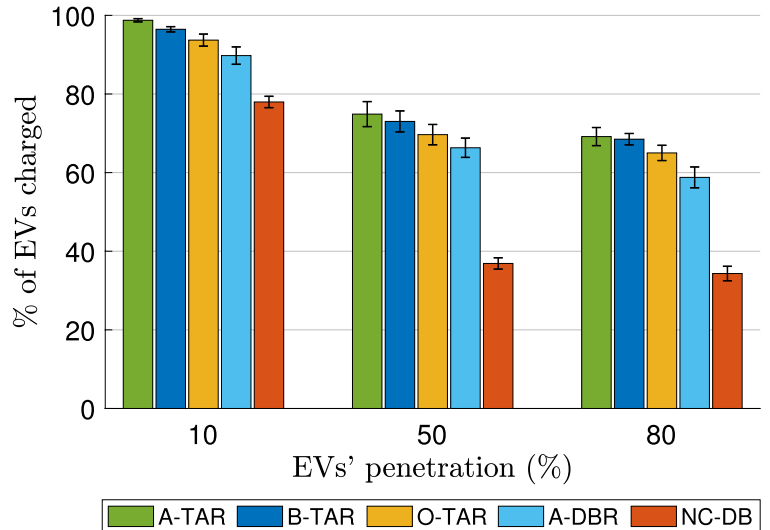

(a)

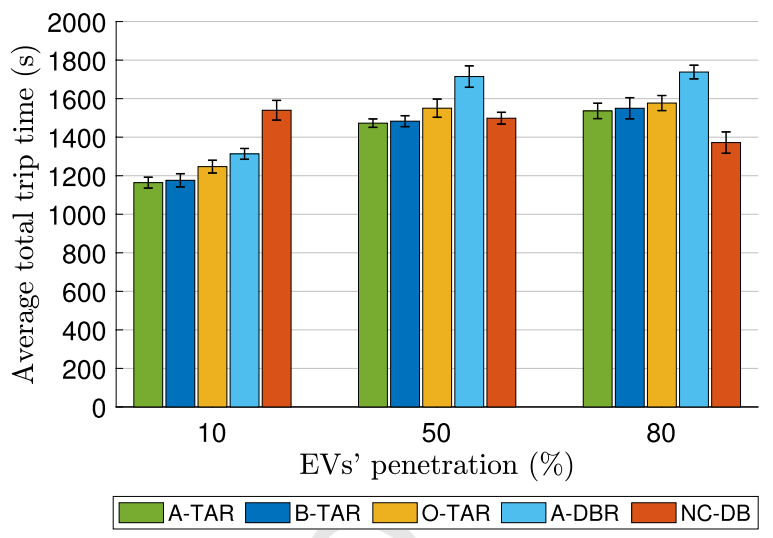

(b)

Fig. 7. Analysis of operating modes (see Table 6). Simulation scenario of a large-size sparse area in Spandau district, Berlin. Six charging slots per CS. (a) \% of charged EVs. (b) Average total trip time till destination. Vehicles' density $\delta_{F F V+E V}=60 \mathrm{veh} / \mathrm{km}^{2}$.

charging stop at a selected CS during the trip. It is calculated based on the average EVs' energy consumption in [48].

- Average service response time: It counts the time interval elapsed from the moment when the EV's battery is below a given threshold, until the moment when the EV receives the information with the selected CS for the EV.

- Communication cost: It evaluates the traffic sent by EVs to the EMMS in terms of service packets per second (pps).

\subsection{Influence of EV penetration}

Fig. 7 shows the percentage of EVs successfully charged among those EVs that required to charge their batteries, i.e. their SoC was not enough to reach destination and was below a threshold So $C_{\text {Threshold. }}$ Thus, the complementary graph depicts the amount of vehicles which required to be charged but did not succeed (i.e., all the CSs where congested). Those vehicles keep looking for an available CS till their batteries get empty, so they never reach their destination. Therefore, their trip times are not included in Figs. 7b and $8 \mathrm{~b}$, where we represent the average total trip time to destination.

From Figs. 7 and 8 we can see that the different EVs charging schemes perform equivalently in both scenarios, Berlin and Barcelona. Notice that the average total trip time to destination (see Figs. $7 \mathrm{~b}$ and $8 \mathrm{~b}$ ) is higher in the Berlin scenario, since the map area is higher and vehicles make longer trips to their destinations.

In Figs. 7a and 8a, we observe that increasing the EV penetration index, the percentage of charged EVs is reduced, whereas the total trip time increases, according to Figs. $7 \mathrm{~b}$ and $8 \mathrm{~b}$. This is because there are more EVs in the scenario and they could need a charging slot sometime during simulation. This fact will increase the chance of having congested CSs.

Our proposal A-TAR achieves the highest percentage of charged EVs, even under a high EVs' penetration percentage (see Figs. 7a and $8 \mathrm{a}$ ). With a $10 \%$ of EVs penetration, A-DBR scheme (which is distance-based) achieves a performance almost same as that obtained by A-TAR or B-TAR. Nonetheless, under higher penetration indexes A-TAR (or B-TAR) scheme clearly outperforms A-DBR scheme. This is because in A-DBR, only distance towards each CS is considered to select the nearest CS. Traffic conditions along the route are not taken into account in A-DBR for the trip time estimation, as it is done in A-TAR and B-TAR. Thus, using A-DBR EVs may not reach the selected CSs at the time they previously reserved. Due to the same reason, the average trip time to destination increases, see Figs. $7 \mathrm{~b}$ and $8 \mathrm{~b}$. This implies that only considering the distance towards each candidate CS in A-DBR, is not a recommended strategy to attain an optimal performance, especially when the EVs' penetration percentage is high. Results for A-DBR show not only a lower number of EVs successfully charged, but also a higher trip time for both simulation scenarios, see (Figs. 7 and 8). Notice that for a fair comparison, both schemes A-TAR and A-DBR implement the same advance communication scheme (see Section 5.3) to manage the charging slot reservations.

With NC-DB, the percentage of successfully charged EVs (out of those EVS which needed to charge their batteries) is dramatically reduced as the EV penetration increases. This is because the simple NC-DB scheme selects a CS, without any reservation, ignoring the status of the other CSs. Hence, it is more probable that an EV selects a CS without any available charging slot (i.e., a congested CS). In spite of that, note that for a penetration index of $80 \%$, NCDB achieves the lowest total trip time (see last column in Figs. 7b and $8 \mathrm{~b}$ ). However, since there is no reservation mechanism, the percentage of charged EVs is very low (see last column in Figs. 7a and $8 \mathrm{a}$ ). The reason is that in those figures we only consider the trip time of vehicles that reached destination, and vehicles that wander looking for an available CS till their batteries get empty are not included.

It is important to highlight the fact that under all the evaluated EVs charging schemes there might happen that: (a) there might be vehicles whose SoC was not enough to reach destination and was below the threshold $S_{0} C_{\text {Threshold }}$ to require a CS in order to charge their batteries; (b) unfortunately, they did not find any free CS in the whole area. In such a case, those vehicles keep trying to find a free CS till their batteries get empty. We have not included their trip times in Figs. $7 \mathrm{~b}$ and $8 \mathrm{~b}$ ), since those figures represent the trip time to destination, and they never reached destination. The chance to happen this situation is considerable higher with NC-DB, since this scheme does not implement any CS reservation.

In Figs. 7 and 8, we observe that O-TAR shows a lower performance than our proposals A-TAR and B-TAR, for low, medium and high EVs' penetration percentages. Particularly, with A-TAR the amount of EVs charged is in average $7 \%$ higher compared to OTAR. The reason is that O-TAR implements an opportunistic routing scheme, where service packets are carried by the EVs instead of being transmitted through the VANET. In case the reservation is not successfully completed (see service messages flow Section 4) at 1-hop, the EV has to wait until another opportunistic encounter with an RSU (i.e., be within an RSU transmission range) takes place. This behavior affects the charging service response time, see Fig. 9a, that increases notably compared to A-TAR and B-TAR. Furthermore, under O-TAR the amount of electricity consumption also increases markedly, see Fig. 9b. This implies that although delay requirements of an EVs charging service may not be stringent (compared to safety applications), if EVs are informed in advance 


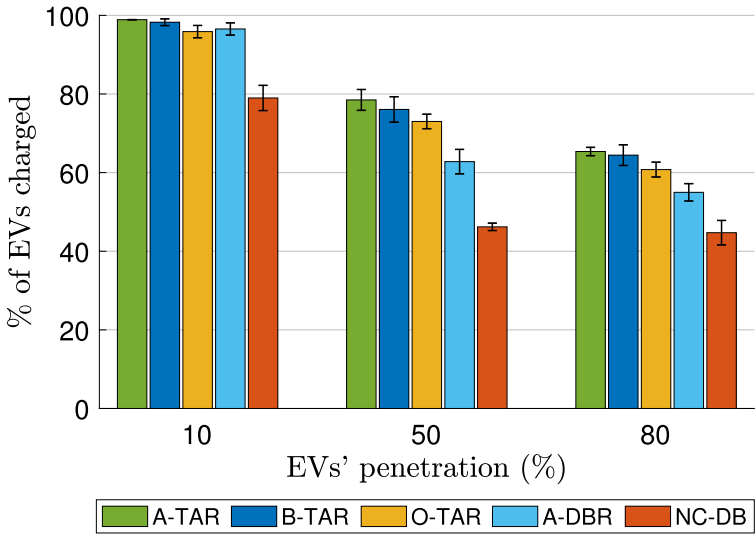

(a)

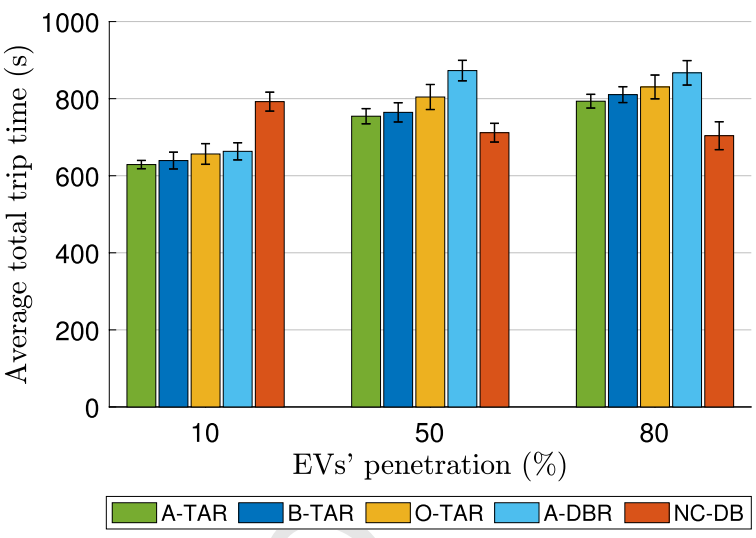

(b)

Fig. 8. Analysis of operating modes (see Table 6). Simulation scenario of a medium-size dense are in Les Corts district, Barcelona. Six charging slots per CS. (a) \% of charged EVs. (b) Average total trip time till destination. Vehicles' density $\delta_{F F V+E V}=120 \mathrm{veh} / \mathrm{km}^{2}$.

about their new destination (i.e., the location of the most suitable CS suggested for battery charging) they will spend less time driving, will use less energy during their driving phase and thus will require less energy during their charging phase.

Concluding, our proposals A-TAR and B-TAR achieve the lowest total trip times to destination among all the reservation-based schemes (A-TAR, B-TAR, O-TAR and A-DBR, see Fig. 8b and Fig. 9b), decrease the amount of energy required by the EV from the CS during the charging phase, and consequently EVs consume a lower amount of energy, see Fig. 9b.

\subsection{Scalability of the routing schemes}

We are interested in evaluating the system scalability in terms of the service communication cost, measuring the traffic generated to provide the proposed charging service. For this, we evaluate our two proposed routing schemes (i.e., Advanced and Basic, see Section 5) under low (60 veh $/ \mathrm{km}^{2}$ ), medium (90 veh $/ \mathrm{km}^{2}$ ), and high (180 veh $/ \mathrm{km}^{2}$ ) vehicles' densities in the simulation scenario of Barcelona city (see Fig. 6a). We compare both proposed routing schemes against the well known GPSR [35] routing protocol as a reference.

In Fig. 10, we observe that the higher the vehicles' density, the better the routing protocols performance. Thus, the performance for $\delta_{E V+F F V}=90 \mathrm{veh} / \mathrm{km}^{2}$ is better than for $\delta_{E V+F F V}=$ $60 \mathrm{veh} / \mathrm{km}^{2}$ next forwarding node selection. This is true up to a maximum vehicle density above which the collision percentage will be too high. Certainly, we observe that for a $\delta_{E V+F F V}=$ $180 \mathrm{veh} / \mathrm{km}^{2}$ the average service packet rate (packets/s) for all the EVs increases again. This is due to higher packet collisions produce more packet re-transmissions. Besides, the Advanced routing scheme shows the highest performance compared with the Basic and GPSR routing protocols, in Fig. 10. The reason is its effectiveness in selecting the next hop to forward packets, since it is based on the efficient multimetric geographic MMMR [33] routing protocol. Here, the GPSR routing protocol generates a higher amount of traffic because of it is not so efficient in the next hop selection to forward service packets and therefore more re-transmissions are required to fulfill the reservation of a charging slot successfully. The Basic routing scheme shows the highest amount of traffic generated (packets/s) mainly due to its flooding-like operation.

\subsection{Discussion on routing schemes}

In Fig. 11 we consider the Barcelona-Les Corts simulation scenario depicted in Fig. 6a, with $80 \%$ EV penetration and a medium

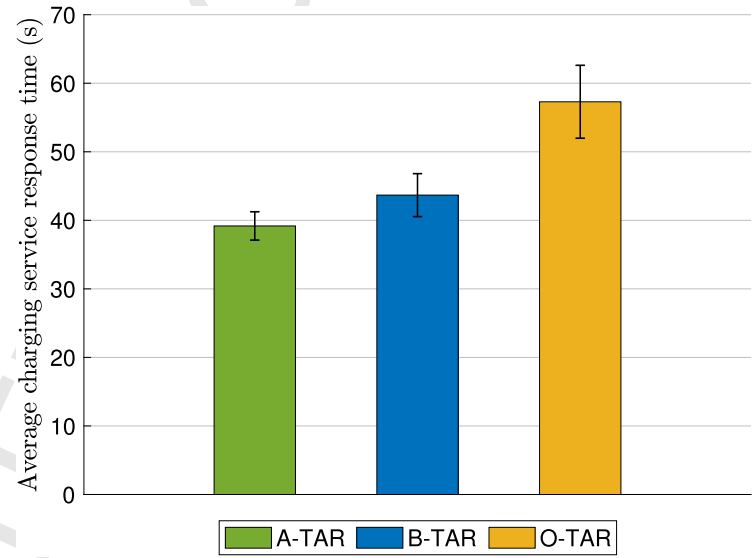

(a)

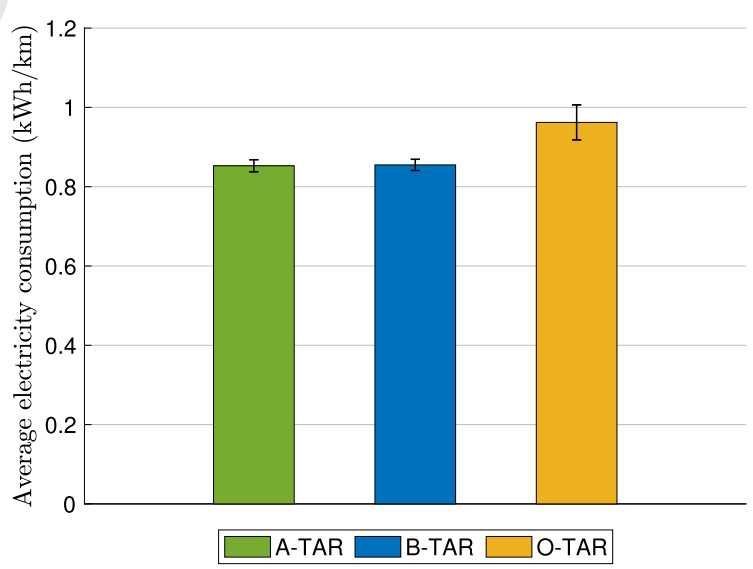

(b)

Fig. 9. (a) Average charging service response time for the three time-based schemes $S R T_{E V}$ in (1). (b) Average electricity consumption per EV.

vehicles' density $\left(\delta_{F F V+E V}=90 \mathrm{veh} / \mathrm{km}^{2}\right)$. In that figure we compare the opportunistic, basic and advance routing schemes in terms of \% of packet losses, average round-trip-time (RTT), and average service response time $S R T_{E V}$ expressed in (1). Default configurations are detailed in Table 5.

In Fig. 11a, we observe that the Opportunistic scheme achieves the lower percentage of packet losses compared with the Basic and Advanced schemes. The reason is that in the Opportunistic 


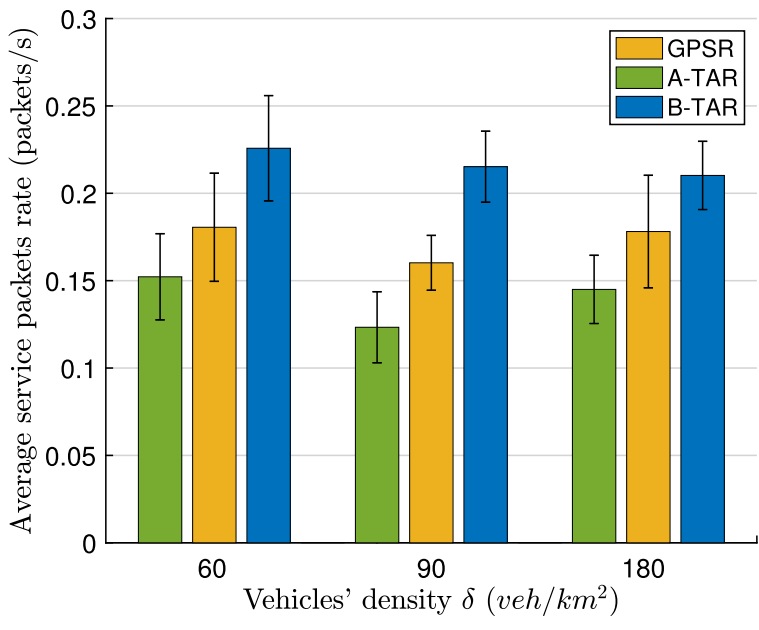

Fig. 10. Average packet rate of the charging service as a function of the total vehicles' density, $\delta_{F F V+E V}$. Simulation scenario of Barcelona city. There are $100 \mathrm{EVs}$.

case, the service message is saved in a local buffer of the energyrequired $\mathrm{EV}$ until an encounter with an RSU happens, i.e. the message is stored and carried by the EV till finding an RSU to deliver it. Conversely, the Advanced scheme selects the best next forwarding node according to a multi-metric score, and stores the service message only in case there is no neighbor to forward the message to. The Basic scheme presents the highest percentage of packet losses because of the flooding-like scheme to forward the EV's service request messages sent at the moment the EV detects a low battery. As we consider a realistic scenario, the energy-requiring EV may remain stopped during a while (e.g., in a red traffic light) without any neighbor around to forward the message. In such a low connectivity case, packet losses will increase.

Fig. 11b shows the average round trip time (RTT) calculated based on those packets that successfully arrived at destination. The RTT counts the time interval elapsed from the moment an EV sends a charging request message to the EMMS, till the reception moment of the correspondent EMMS service response, see RTT Fig. 3. We observe a slightly higher RTT in both proposed schemes (Basic and Advanced) compared with the Opportunistic scheme. The reason is that service messages are forwarded through several hops before reaching their destination (RSU or EV), whereas in Opportunistic the delivery is 1-hop.

Fig. 11c shows the EVs' average service response time $S R T_{E V}$, i.e. the interval of time elapsed from the moment when the EV shows a low battery till the moment when the vehicle receives the answer from the charging service. Despite the good results for the Opportunistic scheme shown in Fig. 11a, and Fig. 11b, Fig. 11c shows that with our two proposed communication schemes (Basic and Advanced) the energy-requesting EV is answered much sooner compared to the Opportunistic case. In Fig. 11c, we observe that the Opportunistic scheme shows the highest delay due to the high amount of time that packets spend in the local buffer of the energy-requesting EV. The average $S R T_{E V}$ in the Basic scheme is higher than with the Advanced scheme because with the Basic scheme more retransmissions are required to complete the charging slot reservation.

Under an ideal channel without errors, the minimum delay (MD) to deliver the packet (VANET transmission over the air in just 1 hop) is given by $M D=\frac{8 \cdot L_{\text {Data }}(\text { bits })}{\text { Throughput(bits/s) }}$. For a nominal data rate of $6 \mathrm{Mbps}$ and a packet length of 200 bytes, the achievable maximum throughput of $802.11 \mathrm{p}$ is around $2 \mathrm{Mbps}$ [49]. The calculated minimum delay, in this case, would be $M D=0.8 \mathrm{~ms}$. In the Opportunistic equivalent case, considering the target urban scenario where vehicles' speed is $S \approx 60 \mathrm{~km} / \mathrm{h}=16.67 \mathrm{~m} / \mathrm{s}$ and the RSU transmission range $R S U_{x}=250 \mathrm{~m}$, a packet would take Oppor $_{\text {Delay }}=\frac{R S U_{x}(m)}{S(m / s)}=16 s$ to get the RSU in 1 hop. These simple numbers help us to realize that forwarding packets through the VANET is always better than store and carry them in the vehicle, since the delay is much lower. This conclusion can also be seen in Fig. 11c, where the average SRT for the Opportunistic case is around 16s, whereas for the Basic scheme is around $6 \mathrm{~s}$ and for the Advanced scheme is about $3 \mathrm{~s}$.

\subsection{Discussion on operating modes}

Given the results obtained for the two different simulation scenarios and considering the EVs' penetration percentage as a major factor to evaluate the usefulness of the operation modes, we can settle that:

1. Low EVs' penetration scenario: Under this configuration, reservation-based schemes (A-TAR, B-TAR, O-TAR and A-DBR) show a similar behavior, see Figs. 7 and 8 . Here, NR-DB (without reservation) can work relatively well in terms of percentage of successfully charged EVs. However, it achieves the highest total trip time, which reduces the EV users' QoE. Besides, the total trip time is remarkable reduced by A-TAR and B-TAR.

2. High EVs' penetration scenario: Under this configuration A-TAR and B-TAR show the best performance even under a high EV penetration (80\%), see Figs. 7 and 8 . Even though in these figures we observe just a subtle improvement of the B-TAR scheme compared to O-TAR, the latter shows a higher average electricity consumption, see Fig. 8a. Here, with NC-DB and A-DBR, the percentage of charged EVs is dramatically reduced and therefore they do not scale well when the number of EV increases.

According to recent studies [2] the EV ratio is foreseen to be $35 \%$ around the year 2022. Rapid battery cost reduction, strong policy support from governments, rising commitment from prime automakers, have put EVs on track to reflect higher sales than fuelpowered vehicles. Indeed, according to [50] EV sales during 2018 have grown around $78 \%$ in China, 34\% in Europe and $79 \%$ in the USA, compared to 2017; and the forecast is that this trend will increase in the coming years. Thus, with the increasing number of EVs in our cities, it will pay-off to arrange a reservation-based energy-charging framework. Results show that our proposals A-TAR and B-TAR provide the best performance even under a high EVs' penetration percentage, in terms of percentage of EVs successfully charged, total trip time to destination, and amount of electricity consumption.

\section{Conclusions and future work}

In this paper, we have proposed an efficient charging management system for on-the-move EVs' charging planning. Our approach includes an advance communication framework based on VANETs with geographical-routing protocols for centralized anticipated charging slot reservations. In specific, we have first introduced a scheme to select the optimal looking to minimize the total charging service time of the energy-requiring EV. The EV's trip time is estimated considering current traffic conditions towards its destination, considering an intermediate charging stop during the trip at the selected CS, leading to a traffic-aware CS selection scheme. Then, we have evaluated the influence of the transmission delay incorporated by opportunistic communication and we demonstrate that our proposed multi-hop multimetric communication scheme can achieve better performance. Evaluation results in the district of Les Corts-Barcelona and Spandau-Berlin have shown the benefits of our proposal, achieving higher percentage of EVs 


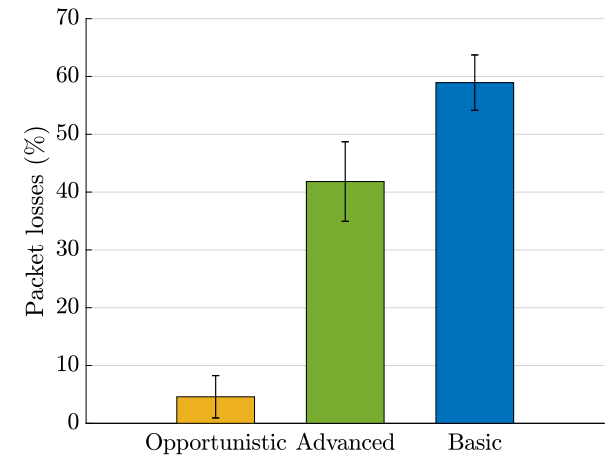

(a)

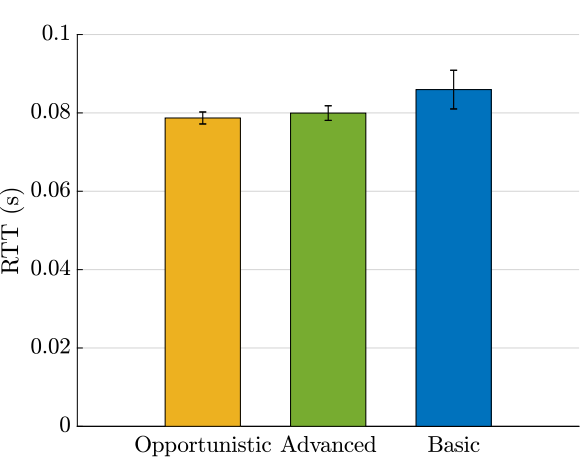

(b)

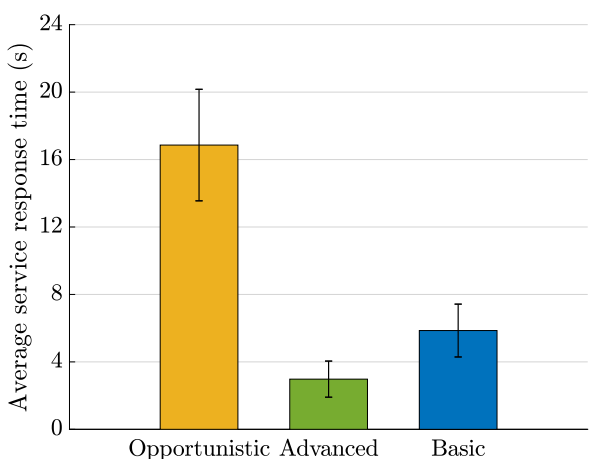

(c)

Fig. 11. Comparison of Opportunistic, Basic and Advanced routing schemes in terms of (a) percentage of packet losses, (b) average round trip time (see RTT in Fig. 3), and (c) average service response time. Simulation scenario of Barcelona city. $80 \%$ EVs' penetration. Vehicles' density $\delta_{E V+F F V}=90 v e h / \mathrm{km}^{2}$. CI $95 \%$.

successfully charged, shorter total trip time to destination, as well as less EVs' energy consumption.

As future work, we plan to analyze the effect of some additional waiting slots arranged in the CSs for those vehicles that arrive at the CS when it is full. Under this scheme, EVs queuing time at CSs should be included in the optimal decision scheme. Also, we plan to include in our proposal a grid-to-vehicle (G2V) communication scheme used to limit the amount of energy to charge each EV as a dynamic percentage of the maximum capacity of its battery, $E V_{\text {max_cap. }}$ That maximum allowed percentage will be computed depending on the SG state. This way, during peak-hours of energy demand in the city, the SG will regulate the amount of energy devoted to the EVs' fleet. Such scheme will need a G2V communication framework to perform efficiently.

We plan to design a machine-learning algorithm o dynamically adjust the coefficients of the model. Finally, in a future work we plan to tackle privacy issues in our proposal of a centralized management system for the EVs charging service. As EVs share information with the EMMS, a secure communication is required to ensure security services (i.e., confidentiality, integrity, and privacy) to protect any personal sensitive information.

\section{Acronyms}

ARS

$\mathrm{CI}$

$\mathrm{C}-\mathrm{V} 2 \mathrm{X}$

CS

CT

DSRC

DBR

DD

EMMS

EV

FFV

G2V

GPSR

ITS

IVC

LoS

MD

MMMR

NC

NNT

OBU

OSM

QoE
Average road speed

Confidence Interval

Cellular vehicle to everything

Charging station

Charging time

Dedicated short range communication

Distance-based reservation

Driving distance

Electro-mobility management server

Electric vehicle

Fossil-fuel vehicle

Grid to vehicle

Greedy perimeter stateless routing

Intelligent transport systems

Inter-vehicle communications

Minimum delay

Multimetric map-aware routing protocol

Non-coordinated

Node neighbors table

On-board units

OpenStreetMaps

Quality of experience
Line of sight
RSU

RTT

SG

SoC

SRT

ST

TAR

TL

TT

V2I

V2G

V2V

$\mathrm{V} 2 \mathrm{X}$

VANET

VDTN

WAVE

WSA

WSM

\section{Declaration of competing interest}

None declared.

\section{References}

[1] L. Canals Casals, E. Martinez-Laserna, B. Amante García, N. Nieto, Sustainability analysis of the electric vehicle use in Europe for $\mathrm{CO}_{2}$ emissions reduction, J. Clean. Prod. 127 (2016) 425-437, https://doi.org/10.1016/j.jclepro.2016.03.120.

[2] ACEA, ACEA position paper reducing $\mathrm{CO}_{2}$ emissions from passenger cars and light commercial vehicles post-2020, Tech. Rep. (May 2016), http://ec.europa. eu/clima/policies/transport/vehicles/cars/index_en.htm.

[3] M. Etezadi-Amoli, K. Choma, J. Stefani, Rapid-charge electric-vehicle stations, IEEE Trans. Power Deliv. 25 (3) (2010) 1883-1887, https://doi.org/10.1109/ TPWRD.2010.2047874.

[4] J1772A: SAE Electric Vehicle and Plug in Hybrid Electric Vehicle Conductive Charge Coupler, SAE International, 2018, https://www.sae.org/standards/ content/j1772_201710/.html.

[5] H. Shareef, M.M. Islam, A. Mohamed, A review of the stage-of-the-art charging technologies, placement methodologies, and impacts of electric vehicles, Renew. Sustain. Energy Rev. 64 (2016) 403-420, https://doi.org/10.1016/j.rser. 2016.06.033.

[6] P. Richardson, D. Flynn, A. Keane, Local versus centralized charging strategies for electric vehicles in low voltage distribution systems, in: 2013 IEEE Power \& Energy Society General Meeting, 2013, p. 1.

[7] S. Habib, M. Kamran, U. Rashid, Impact analysis of vehicle-to-grid technology and charging strategies of electric vehicles on distribution networks - a review, J. Power Sources 277 (2015) 1-486, https://doi.org/10.1016/j.jpowsour.2014.12. 020 .

[8] Z. Hameed Mir, F. Filali, LTE and IEEE 802.11p for vehicular networking: a performance evaluation, EURASIP J. Wirel. Commun. Netw. 2014 (1) (2014) 89, https://doi.org/10.1186/1687-1499-2014-89.

[9] H. Kang, S.H. Ahmed, D. Kim, Y.-S. Chung, Routing protocols for vehicular delay tolerant networks: a survey, Int. J. Distrib. Sens. Netw. 11 (3) (2015) 325027, https://doi.org/10.1155/2015/325027. 
[10] L. Gallo, J. Haerri, Unsupervised long- term evolution device-to-device: a case study for safety-critical v2x communications, IEEE Veh. Technol. Mag. 12 (2) (2017) 69-77, https://doi.org/10.1109/MVT.2017.2669346.

[11] M. Majid, C. Mehmood, U. Farid, S. Ali, S. Anwar, J. Khan, N. Shaukat, Z. Ullah, B. Khan, M. Jawad, A survey on electric vehicle transportation within smart grid system, Renew. Sustain. Energy Rev. 81 (2018) 1329-1349, https://doi.org/10. 1016/j.rser.2017.05.092.

[12] T. Zhang, W. Chen, Z. Han, Z. Cao, Charging scheduling of electric vehicles with local renewable energy under uncertain electric vehicle arrival and grid power price, IEEE Trans. Veh. Technol. 63 (6) (2014) 2600-2612, https://doi.org/10. 1109/TVT.2013.2295591.

[13] R. Li, Q. Wu, S.S. Oren, Distribution locational marginal pricing for optimal electric vehicle charging management, IEEE Trans. Power Syst. 29 (1) (2014) 203-211, https://doi.org/10.1109/TPWRS.2013.2278952.

[14] V. Torres-Sanz, J.A. Sanguesa, F.J. Martinez, P. Garrido, J.M. Marquez-Barja, Enhancing the charging process of electric vehicles at residential homes, IEEE Access 6 (2018) 22875-22888, https://doi.org/10.1109/ACCESS.2018.2829158.

[15] L. Liu, F. Kong, X. Liu, Y. Peng, Q. Wang, A review on electric vehicles interacting with renewable energy in smart grid, Renew. Sustain. Energy Rev. 51 (2015) 648-661, https://doi.org/10.1016/j.rser.2015.06.036.

[16] M. Gharbaoui, L. Valcarenghi, R. Brunoi, B. Martini, M. Conti, P. Castoldi, An advanced smart management system for electric vehicle recharge, in: 2012 IEEE International Electric Vehicle Conference, 2012, pp. 1-8.

[17] I. García-Magariño, G. Palacios-Navarro, R. Lacuesta, J. Lloret, ABSCEV: an agentbased simulation framework about smart transportation for reducing waiting times in charging electric vehicles, Comput. Netw. 138 (2018) 119-135, https:// doi.org/10.1016/j.comnet.2018.03.014.

[18] P. Richardson, D. Flynn, A. Keane, Local versus centralized charging strategies for electric vehicles in low voltage distribution systems, IEEE Trans. Smart Grid 3 (2) (2012) 1020-1028, https://doi.org/10.1109/TSG.2012.2185523.

[19] J. García-Villalobos, I. Zamora, J.I. San Martín, F.J. Asensio, V. Aperribay, Plug-in electric vehicles in electric distribution networks: a review of smart charging approaches, Renew. Sustain. Energy Rev. 38 (2014) 717-731, https://doi.org/10. 1016/j.rser.2014.07.040.

[20] J.C. Mukherjee, A. Gupta, A review of charge scheduling of electric vehicles in smart grid, IEEE Syst. J. 9 (4) (2015) 1541-1553, https://doi.org/10.1109/JSYST. 2014.2356559.

[21] Q. Kang, J. Wang, M. Zhou, A.C. Ammari, Centralized charging strategy and scheduling algorithm for electric vehicles under a battery swapping scenario IEEE Trans. Intell. Transp. Syst. 17 (3) (2016) 659-669, https://doi.org/10.1109/ TITS.2015.2487323.

[22] Y. Cao, N. Wang, Toward efficient electric-vehicle charging using VANET-based information dissemination, IEEE Trans. Veh. Technol. 66 (4) (2017) 2886-2901, https://doi.org/10.1109/TVT.2016.2594241.

[23] S.M.S. Hussain, T.S. Ustun, P. Nsonga, I. Ali, Standard communication based integrated EV charging management in smart grids, IEEE 1609 WAVE and IEC 61850 IEEE Trans. Veh. Technol. 67 (8) (2018) 7690-7697, https://doi.org/10 1109/TVT.2018.2838018

[24] A.H. Abdullah, G. Min, Y. Cao, N. Ahmad, O. Kaiwartya, T. Wang, An EV charging management system concerning drivers' trip duration and mobility uncertainty, IEEE Trans. Syst. Man Cybern. Syst. 48 (4) (2016) 596-607, https://doi.org/10. 1109 /tsmc.2016.2613600

[25] V. Vukadinovic, K. Bakowski, P. Marsch, I.D. Garcia, H. Xu, M. Sybis, P. Sroka, K. Wesolowski, D. Lister, I. Thibault, 3GPP C-V2X and IEEE $802.11 \mathrm{p}$ for vehicleto-vehicle communications in highway platooning scenarios, Ad Hoc Netw. 74 (2018) 17-29, https://doi.org/10.1016/j.adhoc.2018.03.004.

[26] R.A. Johnson, D.W. Wichern, Applied Multivariate Statistical Analysis, 6th edition, Prentice-Hall, Inc., Upper Saddle River NJ, 2007, pp. 360-413.

[27] IBM. Corp. Released, IBM SPSS Statistics for Windows, Version 25.0. Armonk, NY: IBM Corp., Armonk, NY, 2017.

[28] IEEE guide for Wireless Access in Vehicular Environments (WAVE) - architecture, IEEE Std 1609.0-2013 (2014) 1-78, https://doi.org/10.1109/IEEESTD.2014. 6755433.
[29] IEEE standard for Wireless Access in Vehicular Environments (WAVE) - networking services, IEEE Std 1609.3-2016 (2016) 1-160, https://doi.org/10.1109/ IEEESTD.2016.7458115.

[30] J. Liu, J. Wan, Q. Wang, P. Deng, K. Zhou, Y. Qiao, A survey on position-based routing for vehicular ad hoc networks, Telecommun. Syst. 62 (1) (2016) 15-30, https://doi.org/10.1007/s11235-015-9979-7.

[31] G. Karagiannis, O. Altintas, E. Ekici, G. Heijenk, B. Jarupan, K. Lin, T. Weil, Vehicular networking: a survey and tutorial on requirements, architectures, challenges, standards and solutions, IEEE Commun. Surv. Tutor. 13 (4) (2011) 584-616, https://doi.org/10.1109/SURV.2011.061411.00019.

[32] Y. Cao, N. Wang, G. Kamel, Y. Kim, An electric vehicle charging management scheme based on publish/subscribe communic. framework, IEEE Syst. J. 11 (3) (2017) 1822-1835, https://doi.org/10.1109/JSYST.2015.2449893.

[33] C. Tripp Barba, L. Urquiza Aguiar, M. Aguilar Igartua, D. Rebollo Monedero, L.J. de la Cruz Llopis, A.M. Mezher, J.A. Aguilar Calderón, A multimetric, map-aware routing protocol for VANETs in urban areas, Sensors 14 (2) (2014) 2199-2224, https://doi.org/10.3390/s140202199.

[34] A.M. Mezher, M. Aguilar Igartua, Multimedia multimetric map-aware routing protocol to send video-reporting messages over VANETs in smart cities, IEEE Trans. Veh. Technol. 66 (12) (2017) 10611-10625, https://doi.org/10.1109/TVT. 2017.2715719.

[35] B. Karp, H.T. Kung, GPSR: greedy perimeter stateless routing for wireless networks, in: Proceedings of the 6th Annual Int. Conf. on Mobile Computing and Networking, MobiCom '00, ACM, New York, NY, USA, 2000, pp. 243-254.

[36] D. Xiao, L. Peng, C.O. Asogwa, L. Huang, An improved GPSR routing protocol, Int. J. Adv. Comput. Technol. 3 (2011) 132-139, https://doi.org/10.4156/ijact. vol3.issue 5.15.

[37] S. Coast, OpenStreetMap, https://www.openstreetmap.org.

[38] C. Sommer, R. German, F. Dressler, Bidirectionally coupled network and road simulation for improved IVC analysis, IEEE Trans. Mob. Comput. 10 (1) (2011) 3-15, https://doi.org/10.1109/TMC.2010.133.

[39] OMNeT++ discrete event simulator, https://www.omnetpp.org/, 2018

[40] R. Hilbrich, DLR - institute of transportation systems - SUMO simulation of urban MObility, available at http://www.dlr.de/ts/en/desktopdefault.aspx/tabid9883/16931_read-41000/, 2001.

[41] J. Song, Y. Wu, Z. Xu, X. Lin, Research on car-following model based on SUMO, in: Proceedings of 2014 IEEE 7th Int. Conf. on Advanced Infocomm Technology, IEEE/ICAIT 2014, 2015, pp. 47-55.

[42] T. Kurczveil, P. Âlvarez López, E. Schnieder, Implementation of an energy model and a charging infrastructure in SUMO, in: M. Behrisch, D. Krajzewicz, M. Weber (Eds.), Simulation of Urban Mobility, Springer Berlin Heidelberg, Berlin, Heidelberg, 2014, pp. 33-43.

[43] C. Sommer, D. Eckhoff, R. German, F. Dressler, A computationally inexpensive empirical model of IEEE 802.11p radio shadowing in urban environments, in: 2011 8th Int. Conf. on Wireless on-Demand Network Systems and Services, WONS 2011, 2011, pp. 84-90.

[44] E. Standard, ETSI EN 302663 V1.2.1 Intelligent Transport System (ITS); Access layer specification for Intelligent Transport Systems operating in the $5 \mathrm{GHz}$ frequency band 1 (2013) 1-24.

[45] European Parliament and council of the European Union, directive 2014/94/EU of the European Parliament and of the council of 22 October 2014 on the deployment of alternative fuels infrastructure, Official J. Europ. Union L 307 (2014) 1-20 (28.10.2014), https://eur-lex.europa.eu/legal-content/EN/TXT/PDF/ ?uri=CELEX:32014L0094\&from=EN.

[46] Nissan Leaf autonomy and recharge, https://www.nissan.es/vehiculos/nuevosvehiculos/leaf/autonomia.html, 2019.

[47] Tesla Model X owners manual, https://www.tesla.com/sites/default/files/model_ x_owners_manual_europe_es_es.pdf, 2018.

[48] E.V. Charging, How much electricity does an electric car use?, https://www. virta.global/blog.

[49] Y. Wang, X. Duan, D. Tian, G. Lu, H. Yu, Throughput and delay limits of 802.11p and its influence on highway capacity, Proc., Soc. Behav. Sci. 96 (2013) 2096-2104, https://doi.org/10.1016/j.sbspro.2013.08.236

[50] The electric world sales database vehicle, http://www.ev-volumes.com. 


\section{Sponsor names}

Do not correct this page. Please mark corrections to sponsor names and grant numbers in the main text.

SENESCYT, country=Ecuador, grants=
67

68

69

70

71

72

73

74

75

76

77

78

79

80

81

82

83

84

85

86

87

88

89

90

91

92

93

94

95

96

97

98

99

100

101

102

103

104

105

106

107

108

109

110

111

112

113

114

115

116

117

118

119

120

121

122

123

124

125

126

127

128

129

130

131

132 